\title{
THE GEOMETRY OF IDEAL 2-DIMENSIONAL SIMPLICIAL COMPLEXES
}

\author{
CHARALAMPOS CHARITOS ${ }^{1}$ \\ Agricultural University of Athens, Department of Mathematics, 75 Iera Odos, 11855 Athens, Greece \\ e-mail: gmatxax@auadec.aua.gr \\ and ATHANASE PAPADOPOULOS ${ }^{1}$ \\ Institut de Recherche Mathématique Avancée, Université Louis Pasteur et CNRS, 7, rue René Descartes, \\ 67084 Strasbourg, France \\ e-mail: papadopoulos@math.u-strasbg.fr
}

(Received 28 April, 1999)

\begin{abstract}
In this paper, we study geometric structures on 2-dimensional simplicial complexes. In particular, we consider hyperbolic structures and measured foliations on these simplicial complexes. We describe the spaces of such structures and we relate the two resulting spaces in a manner which is analogous to Thurston's compactification of the Teichmüller space of a surface.
\end{abstract}

2000 Mathematics Subject Classification. 57M20, 57M50.

0. Introduction. In this paper, we develop a theory of hyperbolic structures and measured foliations on 2-dimensional simplicial complexes. A 2-dimensional simplicial complex $X$ is a topological space obtained by gluing along their edges a set $\left(T_{i}\right)_{i \in I}$ of topological triangles. A hyperbolic structure on $X$ is a complete metric on the space obtained from $X$ by deleting its vertices, which has the property that each of the triangles $T_{i}$ with its vertices deleted, equipped with the induced metric, is isometric to an ideal hyperbolic triangle in the hyperbolic 2 -space. We call such a structure an ideal hyperbolic structure on $X$.

Given a 2-dimensional simplicial complex $X$ equipped with an ideal hyperbolic structure, we can vary the gluing maps between the ideal hyperbolic triangles. The set of ideal hyperbolic structures is a moduli space, which we call the Teichmüller space $\mathcal{T}(X)$ of the simplicial complex. We study this Teichmüller space and in doing so, we develop coordinates for the Teichmüller space of punctured surfaces which involve shift parameters along a family of disjoint curves whose endpoints are punctures. This is somewhat analogous to the Fenchel-Nielsen coordinates, only there the shifts are along simple closed curves and one needs the lengths of the curves as well. There is a natural topology on the Teichmüller space $\mathcal{T}(X)$ of the simplicial complex. We shall study this topology and we shall construct a compactification of $\mathcal{T}(X)$ by a space whose elements are projective measured foliations on $X$. This will be done in a way which is parallel to the theory of measured foliations on surfaces and of Thurston's compactification of the space of hyperbolic structures on surfaces.

The theory which we develop here will be used for the study of homeomorphisms of 2-dimensional simplicial complexes (see [1]).

\footnotetext{
${ }^{1}$ Work realized under the project No. 41741 between CNRS (France) and NHRF (Greece).
} 
The outline of this paper is the following:

Section 1 contains basic definitions and introductory material on 2-dimensional simplicial complexes and ideal hyperbolic structures on them.

In Section 2, we describe a natural space of parameters for ideal hyperbolic structures on a 2-dimensional simplicial complex $X$ and we define the Teichmüller space $\mathcal{T}(X)$ of $X$.

In Section 3, we study the condition of an ideal hyperbolic structure on $X$ to be complete and we give a formula for the dimension of the Teichmüller space $\mathcal{T}(X)$.

In Section 4, we develop the theory of measured foliations on a 2-dimensional simplicial complex $X$. We define the space $\mathcal{M F}(X)$ of equivalence classes of measured foliations and we study the topology of this space. We describe a natural homeomorphism between the spaces $\mathcal{T}(X)$ and $\mathcal{M F}(X)$.

In Section 5, we embed the two spaces $\mathcal{T}(X)$ and $\mathcal{M F}(X)$ in a functional space $\mathbb{R}_{+}^{\mathcal{S}}$ in such a way that the images of the two spaces are disjoint. The set $\mathcal{S}$ will be a certain set of isotopy classes of arcs in $X$ with fixed endpoints. The embeddings $\mathcal{T}(X) \rightarrow \mathbb{R}_{+}^{\mathcal{S}}$ and $\mathcal{M} \mathcal{F}(X) \rightarrow \mathbb{R}_{+}^{\mathcal{S}}$ provide coordinates for the spaces $\mathcal{T}(X)$ and $\mathcal{M F}(X)$. In the case where $X$ is finite (that is, where $X$ has finitely many cells), the set $\mathcal{S}$ is finite. In the case where the simplicial complex $X$ is a surface (with cusps), these coordinates give new coordinates for the Teichmüller space of a surface with cusps. The embeddings $\mathcal{T}(X) \rightarrow \mathbb{R}_{+}^{\mathcal{S}}$ and $\mathcal{M F}(X) \rightarrow \mathbb{R}_{+}^{\mathcal{S}}$, in the case where the simplicial complex $X$ is finite, are used in Section 6, where we obtain a description of the compactified space $\mathcal{T}(X) \cup \mathcal{P} \mathcal{M F}(X)$ by considering the rays in $\mathbb{R}_{+}^{\mathcal{S}}$ passing through the points of the image of $\mathcal{M F}(X)$ in $\mathbb{R}_{+}^{\mathcal{S}}$, and attaching these rays as points at infinity to the image of $\mathcal{T}(X)$ in $\mathbb{R}_{+}^{\mathcal{S}}$.

We would like to thank the referee for a list of corrections and helpful remarks on a previous version of this paper.

\section{2-dimensional simplicial complexes and ideal hyperbolic structures.}

DeFINITION 1.1. A 2-dimensional simplicial complex is a topological space $X$ equipped with two (finite or infinite) sets $\mathcal{C}$ and $\mathcal{F}$ which satisfy the following five properties:

(i) Each element $T \in \mathcal{C}$ is a topological triangle, that is, a topological space homeomorphic to a 2-dimensional closed disk with three distinguished distinct points on its boundary $\partial T$. The distinguished points are called the vertices of $T$. The closures in $\partial T$ of the connected components of the complement of the vertices are called the edges of $T$.

(ii) Each element $f \in \mathcal{F}$ is a homeomorphism $f: A \rightarrow B$, where $A$ and $B$ are distinct edges of triangles $T$ and $T^{\prime}$ respectively belonging to the collection $\mathcal{C}$ and for each edge $A$ of a triangle $T \in \mathcal{C}$, there exists an $f \in \mathcal{F}$ and an edge $B$ of some triangle $T^{\prime} \in \mathcal{C}$, with $f$ sending $A$ to $B$. We note that it is possible to have $T=T^{\prime}$. The elements of $\mathcal{F}$ are called the gluing maps.

(iii) As a topological space, the simplicial complex $X$ is the quotient of the disjoint union of the elements of $\mathcal{C}$ by the equivalence relation which identifies two edges $A$ and $B$, where $A$ and $B$ are edges of triangles $T$ and $T^{\prime}$ in $\mathcal{C}$, whenever these edges are related by a map $f: A \rightarrow B$ which belongs to the collection $\mathcal{F}$. Let $\pi: \mathcal{C} \rightarrow X$ denote the quotient map. We make the assumption that $\pi$ is injective on each edge of a triangle in $\mathcal{C}$. The image in $X$ by $\pi$ of a triangle $T \in \mathcal{C}$ is called a face of $X$, the image by $\pi$ of an edge of $T$ is called an edge of $X$ and the image by $\pi$ of a vertex of $T$ is called a vertex of $X$. 
(iv) The space $X$ is arcwise connected.

(v) The space $X$ is locally finite around edges, which means that each edge of $X$ is contained in only finitely many faces.

We shall say that a 2-dimensional simplicial complex is finite if its number of faces is finite. We shall require this condition for some of the main results below.

Let $X$ be a 2-dimensional simplicial complex and let $\Lambda \subset X$ be the set of edges of $X$. There is a subset $\Lambda^{\prime} \subset \Lambda$, whose elements are called the singular edges, which are the edges of $X$ whose inverse image by $\pi$ consists in three or more distinct edges of triangles in $\mathcal{C}$. An edge of $X$ which is in $\Lambda^{\prime}-\Lambda$ is called a nonsingular edge.

Let us note that from the topology of $X$, together with the stratification of this space into vertices, edges and faces, we can recover the collection $\mathcal{C}$ of faces of $X$ and the set $\mathcal{F}$ of gluing maps. Indeed, each element $T$ of $\mathcal{C}$, as a topological space, can be defined as the natural completion of the interior of a face of $X$, and the vertices of such a triangle are the inverse images of the vertices in $X$ by the natural map which sends this completion into the space $X$. This defines the set $\mathcal{C}$ of triangles for $X$. In the same way, we can recover the set $\mathcal{F}$ of gluing map by taking the identity maps between pairs of edges of triangles in $\mathcal{C}$ which are mapped to the same edge in $X$. From this observation, we deduce immediately the following

Proposition 1.1. Let $p: \tilde{X} \rightarrow X$ be a covering space and let $X$ be a 2-dimensional simplicial complex. Then $\tilde{X}$ has an induced structure of a 2-dimensional simplicial complex.

Proof. The vertices, edges and faces of $\tilde{X}$ are defined as the inverse images by $p$ of the vertices, edges and faces of $X$ respectively. From these data on $\tilde{X}$, we can recover the collections of triangles and of gluing maps for $\tilde{X}$, as explained above.

Let us recall now some standard notions which will be useful in the sequel. A path in a topological space $E$ is a continuous map $f: I \rightarrow E$, where $I$ is an interval in $\mathbb{R}$. If $I=[a, b]$ is compact and if $x=f(a)$ and $y=f(b)$, then we say that $f$ joins $x$ and $y$. If the interval $I$ is compact, then a path $f: I \rightarrow E$ is also called an arc.

We shall often denote the distance between two points $x$ and $y$ in a metric space by $|x-y|$.

Let $E$ and $E^{\prime}$ be two metric spaces. A map $f: E \rightarrow E^{\prime}$ is said to be isometric if we have $|f(x)-f(y)|=|x-y|$ for every $x$ and $y \in E$. A geodesic in $E$ is a path $g: I \rightarrow E$, which is isometric. If $I=[0, \infty)$ (respectively if $I=\mathbb{R}$ ), then we say that $g$ is a geodesic ray (respectively a geodesic line). A local geodesic in $X$ is a path $g: I \rightarrow E$ such that for each $t \in I$, there exists an interval $I(t) \subset I$ which is a neighborhood of $t$ in $I$, such that the restriction of $g$ to $I(t)$ is geodesic. Let $[a, b]$ be a compact interval of $\mathbb{R}$. The length $\ell(f)$ of an arc $f:[a, b] \rightarrow X$ is defined as $\ell(f)=\sup _{\left(a_{i}\right)} \sum_{i=0}^{n-1}\left|f\left(a_{i}\right)-f\left(a_{i+1}\right)\right|$, where the supremum is taken over all the subdivisions $a=a_{0}<a_{1}<\ldots<a_{n}=b$ of $[a, b]$. The metric space $E$ is said to be geodesic if for all $x$ and $y \in E$, the distance $|x-y|$ is equal to the length of a geodesic arc joining $x$ and $y$. A geodesic segment is the image of a geodesic arc $f:[a, b] \rightarrow X$. If $x=f(a)$ and $y=f(b)$, then we say that the geodesic segment $f([a, b])$ joins $x$ and $y$. By abusing notation, we shall denote by $[x, y]$ a geodesic segment joining $x$ and $y$, even though this segment need not be determined by its endpoints.

A metric space $E$ is said to be a length space if for all $x$ and $y \in E$, the distance $|x-y|$ is equal to the infimum of the lengths of paths joining these points. We note that a geodesic space is a particular case of a length space. 
A triangle $T$ in the metric space $E$ is a triple $x, y, z$ of points in $E$ which are called the vertices of $T$, together with a triple $[x, y],[x, z],[y, z]$ of geodesic segments joining them. The geodesic segments are called the sides of $T$.

Let $\mathbb{H}^{2}$ be the hyperbolic plane. If $T$ is a triangle in $E$, then a comparison triangle for $T$ in $\mathbb{H}^{2}$ is a triangle $T^{*}$ in $\mathbb{H}^{2}$ whose sides have the same lengths as those of the sides of $T$, together with a map $f$ from the disjoint union of the sides of $T$ onto the sides of $T^{*}$, such that $f$ is consistent on the vertices on $T$ and such that the restriction of $f$ to each side of $T$ is an isometry. The map $f$ is called a comparison map for $T$. We note that the comparison triangle $T^{*}$ together with its associated comparison map $f$ are unique up to post-composition by an isometry of $\mathbb{H}^{2}$.

A triangle $T$ in $E$ is said to satisfy the CAT(-1)-inequality if its associated comparison map $f$ is distance non-decreasing, that is, if we have, for every $x$ and $y$ in $T$,

$$
|x-y|_{E} \leq|f(y)-f(x)|_{\mathbb{Q}^{2}} .
$$

A metric space $E$ is said to be a CAT(-1)-space if every triangle in $E$ satisfies the CAT(-1)-inequality. A metric space $E$ is said to be a local CAT(-1)-space if every point in $E$ has a neighborhood which is a CAT(-1)-space with respect to the induced metric.

Let $\partial \mathbb{H}^{2}$ be the boundary at infinity of $\mathbb{H}^{2}$. An ideal hyperbolic triangle is a subset of $\mathbb{H}^{2}$ defined as the convex hull of three distinct points in $\partial \mathbb{H}^{2}$. By forgetting the hyperbolic geometry, an ideal hyperbolic triangle is in particular a topological triangle (in the sense given in Definition 1.1. (i)) with its vertices deleted.

Let $X$ be a 2-dimensional simplicial complex, let $\mathcal{C}$ be its set of topological triangles, let $\mathcal{F}$ be its set of gluing maps and let $S$ be its set of vertices. We shall be interested in metrics on $X-S$ which are obtained in the following manner. For each topological triangle $T \in \mathcal{C}$, we delete the vertices of $T$ and we equip this triangle with its vertices deleted with a metric which makes it isometric to an ideal hyperbolic triangle. In this way each side of $X$ (with its vertices deleted) is equipped with an induced metric and becomes isometric to the infinite real line. We take now each map $f: A \rightarrow B$ in $\mathcal{F}$ to be an isometry, with respect to these metrics. Each face of $X$, with its vertices deleted, is then equipped with the metric induced from that of the corresponding triangle in $\mathcal{C}$, and it becomes in this way isometric to an ideal hyperbolic triangle. The length $\ell_{X}(\gamma)$ of a path $\gamma: I \rightarrow(X-S)$ is defined now as the sum of the lengths of the components of the intersection of $\gamma$ with the faces of $X$. Let $h:(X-S) \times(X-S) \rightarrow \mathbb{R}$ be the function defined in the following way: for $(x, y) \in(X-S) \times(X-S), h(x, y)$ is equal to the infimum of the set of lengths of paths in $X-S$ joining these points.

Proposition 1.2. The function $h:(X-S) \times(X-S) \rightarrow \mathbb{R}$ is a metric on $X-S$, and the metric space $(X-S, h)$ is a length space.

Proof. It is clear from the definition that $h$ satisfies all the properties of being a metric, except perhaps the non-degeneracy property, that is, the property which says that $h(x, y)=0 \Rightarrow x=y$. To prove this last property, we choose a collection $\left\{B\left(z, r_{z}\right)\right\}_{z \in X}$, with $r_{z}>0$ for each $z \in X$, and where each $B\left(z, r_{z}\right)$ is defined as the set $\left\{w \in X, h(z, w)<r_{z}\right\}$. (This is the "open ball" centred at $z$ of radius $r_{z}$, for the, up to now, pseudo-metric $h$.) To prove that $h(x, y)=0 \Rightarrow x=y$ for every $x$ and $y$ in $X$, it is sufficient to prove this property for each given $z \in X$ and for each $x$ and $y$ 
contained in the set $B\left(z, r_{z}\right)$. In other words, it is sufficient to show that $B\left(z, r_{z}\right)$ is in fact a metric space. Let us fix such a set $B\left(z, r_{z}\right)$. If $z$ is in the interior of a face of $X$ (respectively if $z$ is on a nonsingular edge), then we can choose $r_{z}$ to be small enough so that the set $B\left(z, r_{z}\right)$ is contained in this face (respectively, in a union of the two faces of $X$ which are glued along that common edge). The result is clear in this case since $B\left(z, r_{z}\right)$ is isometric to an open ball in $\mathbb{M}^{n}$, which implies in particular that this set is a metric space. In the case where $z$ is on a singular edge, then, since $X$ is locally finite, we can take $r_{z}$ small enough so that the set $B\left(z, r_{z}\right)$ is the union of a finite number of half-open disks (that is, open disks cut open along their diameters) in $\mathbb{H}^{2}$, which have diameters of equal lengths and which are glued along these diameters. With this description, it is clear that in this case also, $B\left(z, r_{z}\right)$ is a metric space. This shows that $h$ is a metric. The fact that this metric space is a length space is clear from the definition of the metric.

We shall call the metric $h$ on $X-S$ the canonical metric associated to the given data (that is, to the 2-dimensional simplicial complex $X$ together with the structure of ideal triangles on the topological triangles of $X$ and the choice of the gluing maps between the edges of the ideal triangles). Any such metric $h$ will be called an ideal hyperbolic structure on $X$, and we shall say that the space $X$, equipped with $h$, is an ideal 2-dimensional simplicial complex. We shall always assume that the metric $h$ on $X-S$ is complete. We shall talk also (by abuse of language) of a metric on $X$, although the metric is defined strictly speaking on $X-S$. (In the space $X$, the distance from any point to the vertex of an ideal triangle is infinite.)

Proposition 1.3. Let $X$ be an ideal 2-dimensional simplicial complex and let $S$ be the set of vertices of $X$. Then $X-S$ is a geodesic metric space and each point in $X-S$ has a neighborhood which is isometric to the union of a finite number of half-disks in $\mathbb{H}^{2}$ whose diameters have equal lengths, the half-disks being glued along their diameters.

Proof. That the last statement is true was already observed in the proof of Proposition 1.2, and the result stating that the metric space $X$ is geodesic follows from a theorem of Cohn-Vossen which asserts that a complete locally compact length space is geodesic (see [5], Chapter 1, Theorem 1.10).

Proposition 1.4. Let $X$ be an ideal 2-dimensional simplicial complex, with $S$ its set of vertices. Then $X-S$ is a local CAT(-1)-space.

Proof. Let $x$ be an arbitrary point of $X$, and take a neighborhood of $x$ in $X$ which is obtained by gluing a finite number of half-disks in $\mathbb{H}^{2}$ along these diameters. (Note that if $x$ is either in the interior of a face of $X$, or is on a nonsingular edge of $X$, then this finite number is equal to 2.) Each of these half-disks is a CAT(1)-space, and the proof of the proposition follows therefore from the fact that the space obtained by gluing a finite number of CAT(-1)-spaces along convex subsets is a CAT(-1)-space (see [4], Ch. 10 Corollary 5 and Lemma 9).

COROLlary 1.5. Let $X$ be an ideal 2-dimensional simplicial complex, with $S$ its set of vertices. If $X-S$ is simply connected, then $X-S$ is a CAT(-1)-space. 
Proof. This follows from the fact that a simply connected local CAT(-1)-space is a CAT(-1)-space (see [4], Chapter 3, Theorem 12).

The following two corollaries are also consequences of basic results on CAT(1)-spaces (see [4], Chapter 10, Theorem 13):

COROllary 1.6. Let $X$ be an ideal 2-dimensional simplicial complex, and let $S$ be its set of vertices. If $x$ and $y$ are two arbitrary points in $X-S$, then each homotopy class of curves in $X-S$ joining the points $x$ and $y$ contains exactly one local geodesic path. In the case where $X-S$ is simply connected, there is a unique geodesic arc (up to parametrization) in $X-S$ joining $x$ and $y$.

COROllary 1.7. Let $X$ be an ideal 2-dimensional simplicial complex, let $S$ be its set of vertices, and suppose that $X-S$ is simply connected. Then $X-S$ is contractible.

The following result will also be useful:

Proposition 1.8. Let $X$ be an ideal 2-dimensional simplicial complex, with $S$ its set of vertices. Suppose that each edge of $X$ is adjacent to exactly two faces and that $X-S$ is simply connected. Then $X-S$ is isometric to the 2-dimensional hyperbolic space $\mathbb{H}^{2}$.

Proof. The space $X-S$ is a simply connected surface equipped with a complete Riemannian metric of constant curvature -1 . By the uniformization theorem, $X-S$ is isometric to $\mathbb{H}^{2}$.

Proposition 1.9. Let $X$ be an ideal 2-dimensional simplicial complex and let $S$ be its set of vertices. Suppose that each edge of $X$ is adjacent to exactly two faces. Let $X^{\prime} \subset X-S$ be the union of finitely many faces, and suppose that $X^{\prime}$ is connected. Then $X^{\prime}$ is isometric to a finite ideal polygon in $\mathbb{H}^{2}$.

Proof (Sketch). We start with an arbitrary face of $X^{\prime}$ and we send it by an isometry $f$ to an ideal hyperbolic triangle in $\mathbb{H}^{2}$. Using induction, we extend $f$ to nearby faces and we obtain a map from $X^{\prime}$ to $\mathbb{H}^{2}$ which is an isometry onto an finite ideal polygon.

Proposition 1.10. Let $X$ be an ideal 2-dimensional simplicial complex with $S$ its set of vertices, and suppose that $X-S$ is simply connected. Then every edge of $X$ is the image of a geodesic line.

Proof. One way to prove the proposition is to note that $X-S$ is a global CAT(-1)space, and then that each local geodesic in this space is a geodesic. But we can also give a direct proof of this proposition as follows.

Let $\lambda \subset X$ be an edge of $X$. To prove that $\lambda$ is the image of a geodesic line, it suffices to prove that if $x$ and $y$ are points on $\lambda$ and if $g:[a, b] \rightarrow X$ is a geodesic joining them, then the image $[x, y]$ of $g$ is contained in $\lambda$. Suppose for contradiction that $[x, y]$ is not contained in $\lambda$. Then $g$ is transverse to $\lambda$, and to all the other edges of $X$. Consider the sequence of consecutive faces of $X$ that have nonempty intersection with the segment $[x, y]$, starting at $x$. Since $X$ is simply connected, Proposition 
1.9 implies that the union of these faces is homeomorphic to a finite ideal polygon in $\mathbb{H}^{2}$ which is embedded in $X$. This implies that $x$ and $y$ cannot be on the same edge of $X$. This proves Proposition 1.10.

2. The Teichmüller space of a 2-dimensional simplicial complex. Let $T$ be an ideal hyperbolic triangle. Then $T$ has a distinguished point, called its centre. This point can be defined as the unique fixed point of the two order-3 symmetries of $T$ which send an edge to an adjacent edge. Furthermore, each edge of $T$ is equipped with a distinguished point, which is the foot of the perpendicular drawn from the centre of $T$ to that edge. We shall call this point the centre of the edge. In Figure 1, an ideal triangle is represented in the upper half-space model of $\mathbb{U}^{2}$, together with the three perpendiculars from the centre of the triangle to the centres of the edges. The point $p$ is the centre of the triangle and $p_{1}, p_{2}$ and $p_{3}$ are the centres of the edges.

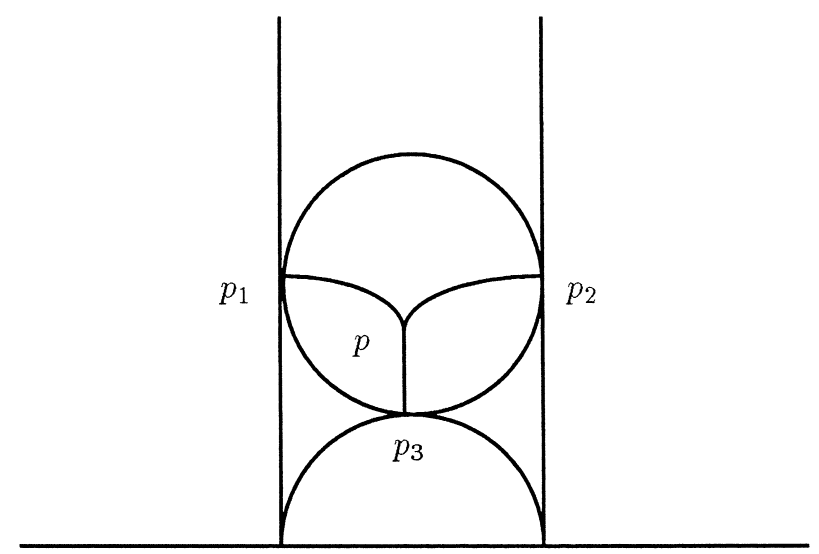

Figure 1

Definition 2.1. Let $X$ be a 2-dimensional simplicial complex, with $\mathcal{C}$ its set of triangles and $\mathcal{F}$ the associated set of gluing maps. Two ideal hyperbolic structures $h$ and $h^{\prime}$ on $X$ are said to be equivalent if there exists a homeomorphism $F: X \rightarrow X$ which induces the identity map on the edges of $X$ and which satisfies $F^{*}(h)=h^{\prime}$. We denote by $\mathcal{T}(X)$ the set of equivalence classes of ideal hyperbolic structures on $X$ and we call this set the Teichmüller space of $X$.

Let $X$ be a 2-dimensional simplicial complex equipped with an ideal hyperbolic structure $h$ and let $S$ be the set of vertices of $X$. We equip each edge of $X$ with an orientation. The edges of the triangles in $\mathcal{C}$ are then equipped with an induced orientation, via the map $\pi: \mathcal{C} \rightarrow X$. Each edge is equipped also with a metric, which is induced from the ideal hyperbolic structure $h$ on $X$. Furthermore, each edge $A$ of a triangle in $\mathcal{C}$, being naturally the edge of an ideal hyperbolic triangle, has a distinguished point. The orientation, the distinguished point and the metric on $A$ make that edge canonically isometric to the real line, and therefore they induce a canonical system of coordinates on that edge. In this way, each map $f: A \rightarrow B$ in $\mathcal{F}$ is an orientation preserving isometry of $\mathbb{R}$ and is therefore determined by a unique real 
number defined as the algebraic measure of the image $f(a)$ of the distinguished point $a$ of $A$, with respect to the coordinate system of $B$. We shall denote this algebraic measure by $x_{h}(f)=x(f)$. For each $f \in \mathcal{F}$, we call the value $x(f)$ the shift, or the shift parameter of the isometry $f$. We obtain in this way a map $\mathcal{I}: \mathcal{T}(X) \rightarrow \mathbb{R}^{\mathcal{F}}$, which is defined by the formula

$$
\mathcal{I}(h)(f)=x_{h}(f)
$$

for every $h \in \mathcal{T}(X)$ and for every $f \in \mathcal{F}$.

The map $\mathcal{I}$ is clearly injective, since each element of $\mathcal{T}(X)$ is determined by the associated set of gluing maps, which are in this case isometries between edges of ideal hyperbolic triangles.

We equip the set $\mathcal{F}$ with the discrete topology, $\mathbb{R}^{\mathcal{F}}$ with the product topology and the Teichmüller space $\mathcal{T}(X)$ with the topology induced from the embedding $\mathcal{I}: \mathcal{T}(X) \rightarrow \mathbb{R}^{\mathcal{F}}$. Let us note that the map $\mathcal{I}$ is not necessarily onto, because of the requirement that an ideal hyperbolic structure on $X$ is complete. In Section 3 below, we shall consider the issue of completeness and we shall see that this condition is given by a set of linear equations in $\mathbb{R}^{\mathcal{F}}$. This will imply that the space $\mathcal{T}(X)$ is homeomorphic to a product space $\mathbb{R}^{n}$ (with $n$ finite or infinite).

Proposition 2.2. Let $X$ be a 2-dimensional simplicial complex and let $p: \tilde{X} \rightarrow X$ be a covering space of $X$. There is a natural embedding $u: \mathcal{T}(X) \rightarrow \mathcal{T}(\tilde{X})$.

Proof. By Proposition 1.1, $\tilde{X}$ has a natural structure of a 2-dimensional simplicial complex. Let $S$ (respectively $\tilde{S}$ ) be the set of vertices of $X$ (repectively $\tilde{X}$ ). An ideal hyperbolic structure $h$ on $X$ is a metric on $X-S$ and by lifting this metric to $\tilde{X}-\tilde{S}$ we obtain an ideal hyperbolic structure on $\tilde{X}$. Let $u: \mathcal{T}(X) \rightarrow \mathcal{T}(\tilde{X})$ be the resulting map. The values of the shift parameters associated to the gluing maps of the simplicial complex $\tilde{X}$ are equal to the values of the shift parameters of the corresponding gluing maps of $X$. This implies that the map $u$ is continuous and injective. This proves the proposition.

3. The completeness of ideal hyperbolic structures and the dimension of the Teichmüller space of a finite 2-dimensional simplicial complex. In this section, we study the condition on the shift parameters of an ideal hyperbolic structure on a 2dimensional simplicial complex $X$ which is imposed by the fact that this structure is complete. We use this to obtain a formula for the dimension of the Teichmüller space $\mathcal{T}(X)$ of $X$ in the case where $X$ is a finite 2-dimensional simplicial complex, that is, when number of faces of $X$ is finite. (Of course, in the case where the number of faces is infinite, the dimension of $\mathcal{T}(X)$ is infinite.)

We start with the following well-known proposition, which we shall generalize below to include the case of ideal 2-dimensional simplicial complexes.

Proposition 3.1. Let $M$ be a surface obtained from a closed surface by removing a nonempty finite set of points. Let $h$ be a hyperbolic structure of finite volume on $M$. Then, the structure $h$ is complete if and only if one of the following two equivalent conditions holds: 
(i) Around each puncture of $M$, there is a foliation by closed horocycles (that is, the leaves of the foliation are closed and they lift to the universal covering of $M$ as pieces of horocycles).

(ii) Each puncture of $M$ is a cusp. In other words, the puncture, regarded as an ideal vertex, has a neighborhood isometric to the quotient of a region $\{y \geq a\}$ of the upper-half-plane model of $\mathbb{H}^{2}$ (where a is a positive real number), by the translation $z \mapsto \rightarrow z+1$.

Proof. See [8], Chapter 3, Proposition 3.4.18.

Let $X$ be now a finite 2-dimensional simplicial complex, let $\mathcal{C}$ and $\mathcal{F}$ denote respectively, as in Definition 1.1 above, the sets of topological triangles and of gluing maps of $X$ and let $\pi: \mathcal{C} \rightarrow X$ be the natural quotient map.

In the case where $X$ is a surface (that is, in the case where $X$ has no singular edges at all), then if $X$ is equipped with an ideal hyperbolic structure $h$, the fact that this structure is complete, together with the fact that $X$, being locally finite, has only finitely many triangles having a common vertex, imply that each vertex of $X$ is a cusp in the usual sense of 2-dimensional hyperbolic geometry (see case (ii) of Proposition 3.1.). More generally, if $X$ is a 2-dimensional simplicial complex equipped with an ideal hyperbolic structure $h$ and if $v$ is a vertex of $X$, then we shall call the vertex $v$ a cusp of $X$.

Let us study more closely the constraints on the ideal hyperbolic structure on a 2-dimensional simplicial complex which are implied by the fact that this structure is complete.

Let $X$ be again a 2-dimensional simplicial complex (without any ideal hyperbolic structure) and let $v$ be a vertex of $X$. We can represent the link of $v$ in $X$ by a simplicial graph $\Gamma(v) \subset X$, obtained in the following manner: consider a small closed ball neighborhood $B(v)$ centred at $v$ (the ball being defined with respect to any simplicial metric). We take then, to define $\Gamma(v)$, one vertex on each half-edge of $X$ abutting on $v$, and we join two such vertices by an edge if and only if these vertices corespond to half-edges which are on the boundary of a face of $X$. Since $X$ is locally finite, the graph $\Gamma(v)$ is finite. The closed ball $B(v)$ has a natural structure of a cone of the form $v . \Gamma(v)$, that is, the topological quotient space $[0,1] \times \Gamma(v) / \sim$, where $\sim$ is the equivalence relation identifying the subspace $\{0\} \times \Gamma(v)$ to a point.

Let us fix now an ideal hyperbolic structure $h$ on $X$ and let $v$ be again an arbitrary vertex (which, according to our definition, is a cusp) of $X$ and let $\Gamma(v)$ be its associated simplicial graph. Let $L$ be a simplicial simple closed curve embedded in $\Gamma(v)$ and let us fix an orientation on $L$.

Let $\left\{z_{1}, \ldots, z_{n}\right\}$ be the sequence of vertices of $\Gamma(v)$ which are on $L$, with the cyclic order preserved. Each vertex $z_{i}$ is associated to a half-edge $e_{i}$ of $X$ ending at $v$. We equip each $e_{i}$ with the orientation directed towards the vertex $v$. There is a homeomorphism $f_{i} \in \mathcal{F}, f_{i}: A_{i} \rightarrow B_{i}$, where $A_{i}$ and $B_{i}$ are two edges of triangles of $\mathcal{C}$, such that the image $\pi\left(A_{i}\right)=\pi\left(B_{i}\right)$ contains the half-edge $e_{i}$. We saw in Section 2 above that the hyperbolic structure $h$, together with the orientation on the edges $A_{i}$ and $B_{i}$ induced from that of $e_{i}$, induce a well-defined coordinate systems on $A_{i}$ and $B_{i}$. We define $x\left(z_{i}\right)$ as the shift parameter $x\left(f_{i}\right)$, that is, the algebraic measure of the image $f\left(a_{i}\right)$ of the origin of $A_{i}$ with respect to the coordinate system of $B_{i}$.

Thus, we have a real parameter $x\left(z_{i}\right)$ associated to every vertex $z_{i}(i=1, \ldots, n)$. The subset of $v . \Gamma(v)$ corresponding to the curve $L$ has naturally the structure of a 
cone v.L. The fact that the ideal hyperbolic structure $h$ on $X$ is complete implies that the subset $v . L-\{v\}$ of $X$ is isometric to a cusp in the usual sense (that is, a cusp in a hyperbolic surface). Therefore, by Proposition 3.4.18 of [8], the parameters $x\left(z_{i}\right)$ satisfy the relation

$$
x\left(z_{1}\right)+\ldots+x\left(z_{n}\right)=0 .
$$

There is one such relation for each cusp $v$ of $X$ and for each choice of simple closed curve $L$ in the simplicial graph $\Gamma(v)$. We have now the following:

Proposition 3.2. Let $X$ be a 2-dimensional simplicial complex and let $S$ be its set of vertices. Suppose that we have a hyperbolic metric $h$ on $X-S$, satisfying all the properties of an ideal hyperbolic structure, except perhaps the property which makes it a complete metric. Then $h$ is complete if and only if for every vertex $v$ of $X$ whose link is represented by a simplicial graph $\Gamma(v)$, and for every simplicial simple closed curve $L \subset \Gamma(v)$ whose vertices are denoted by $z_{1}, \ldots, z_{n}$, the relation $(*)$ above is satisfied.

Proof. Consider the compact cone neighborhood $v . \Gamma(v)$ of $v$ in $X$. We equip the set $v . \Gamma(v)-v$ with the hyperbolic metric induced by $h$. Let $L \subset \Gamma(v)$ be a simplicial simple closed curve. Then, the cone v.L is a surface which is homeomorphic to a closed disk, and $v . L-v$ is homeomorphic to a cylinder. There is a hyperbolic structure on this cylinder, induced by $h$. It is clear that the metric on $v \cdot \Gamma(v)-v$ is complete if and only if the metric on each v.L $-v$ is complete. By Proposition 3.1, the metric on $v . L-v$ is complete if and only if the relation (*) associated to $L$ is satisfied.

It is easy now to compute the number of independent parameters which determine the dimension of the Teichmüller space $\mathcal{T}(X)$. Let $v_{1}, \ldots, v_{n}$ be the vertices of $X$, with $\Gamma_{i}=\Gamma\left(v_{i}\right)$ the link of $v_{i}$ for each $I=1, \ldots, n$. Each vertex $v_{i}$ has a neighborhood in $X$ homeomorphic to the cone $v_{i}$. $\left(v_{i}\right)$. Let $r_{i}$ be the rank of the free group $\pi_{1}\left(\Gamma_{i}\right)$. The parameters $x\left(z_{i}\right)$ defined above satisfy $r$ independent relations of the form $(*)$, with $r=\sum_{i=1}^{n} r_{i}$. We have therefore the following

Theorem 3.3. Let $X$ be a finite 2-dimensional simplicial complex, having $n$ vertices $v_{1}, \ldots, v_{n}$, such that for every $i=1, \ldots, n$, a neighborhood of the vertex $v_{i}$ is the cone over a graph $\Gamma_{i}$ such that the rank of $\pi_{1}\left(\Gamma_{i}\right)$ is equal to $r_{i}$. Let $N$ be the number of independent gluing maps $f: A \rightarrow B$ that appear in the definition of $X$. Then, we have

$$
\operatorname{dim} \mathcal{T}(X)=N-\sum_{i=1}^{n} r_{i}
$$

4. Measured foliations on 2-dimensional simplicial complexes. In this section, we define measured foliations on 2-dimensional simplicial complexes and we study the space $\mathcal{M F}(X)$ of measured foliations on a given 2-dimensional simplicial complex $X$. The theory is parallel to that of ideal hyperbolic structures on $X$, and several results on the space $\mathcal{M F}(X)$ are, formally, analogues of those on the Teichmüller space $\mathcal{T}(X)$. We shall use these facts in the next section to relate in a natural geometric manner the two spaces. 
Definition 4.1. Let $X$ be a 2-dimensional simplicial complex. A measured foliation $F$ on $X$ is a structure whose restriction to each face of $X$ is a singular 1dimensional foliation, satisfying the following five properties:

(i) The foliation $F$ has a transverse invariant measure which is equivalent to Lebesgue measure. (This implies in particular that on each arc transverse to the leaves, the induced measure is of full support.)

(ii) The foliation $F$ restricted to each face of $X$ is transverse to the boundary of that face and it has one singular point in the interior of that face. The topological picture at the singular point is given in Figure 2, and this type of singularity is called a tripod.

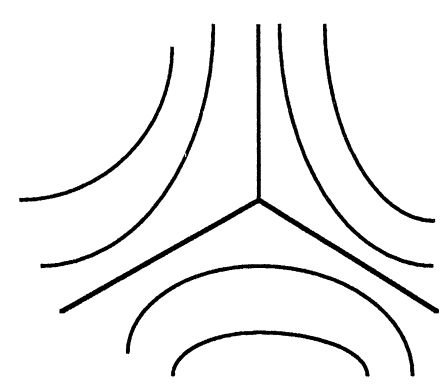

Figure 2

(iii) On each edge of $X$, the transverse measures coming from all the adjacent faces coincide.

(iv) The total transverse measure of each edge of $X$ is infinite in both directions.

(v) The foliation $F$ is trivial around each vertex of $X$. This condition means the following:

Definition 4.1.1. Let $v$ be a vertex of $X$. The foliation $F$ is said to be trivial around $v$ if there exists a neighborhood $N$ of $v$ in $X$ such that the restriction $F_{\mid N}$ of $F$ to $N$ has the following topological structure: Consider an abstract finite simplicial graph $L(v)$, and consider the product space $L(v) \times[0,1)$. Let $F^{\prime}(v)$ be a foliation of this product space whose leaves are the fibres of the projection onto the second factor. The structure of the foliated neighborhood $N$ is such that there exists a simplicial graph $L(v)$, equipped with its foliation $F^{\prime}(v)$, such that $N$ is homeomorphic to the quotient space $(L(v) \times[0,1)) / \sim$, where $\sim$ is the equivalence relation which identifies the whole subspace $L(v) \times\{0\}$ to a point, and such that this point is the image of the vertex $v$ by the quotient map $(L(v) \times[0,1)) \rightarrow(L(v) \times[0,1)) / \sim$. The foliation $F_{\mid N}$ is the induced foliation on the quotient space. Note that in the case where the neighborhood $N$ is a 2-dimensional disk with $v$ being an interior point of that disk, then the foliation $F_{\mid N}$ is a foliation by closed leaves, with $v$ being a singular point whose local model is that of a "centre".

We note the following equivalent formulation of Definition 4.1.1. For every vertex $v$ of $X$, we know, from the fact that the triangulation is locally finite, that there are only finitely many triangles having $v$ as a vertex. Consider the link of the vertex $v$ and represent it, as in Section 3 above, by a finite simplicial graph $\Gamma(v) \subset X$. For every simplicial simple closed curve $L \subset \Gamma(v)$, let $X(L)$ be the union of the triangles in $X$ which are associated to the edges of the simplicial curve $L$. The space $X(L)$, intersected with any small closed ball neighborhood of $v$ (the ball being taken 
with respect to an arbitrary simplicial metric on $X$ ), is a surface equipped with a foliation $F(L)$, induced by $F$. The foliation $F$ is then trivial around the vertex $v$ if for every $L$, the foliation $F(L)$, in a small ball around $v$, is trivial, that is a foliation of a disk by closed leaves around a singular point whose type is a centre, this centre being the vertex $v$.

We shall say that two measured foliations on $X$ are equivalent if they differ by an isotopy which preserves the transverse measure and which preserves the sets of vertices, of edges and of triangles of $X$. We denote by $\mathcal{M F}(X)$ the set of equivalence classes of measured foliations.

Proposition 4.2. Let $F$ be a measured foliation on $X$ and let $s$ be the homotopy class of an arc in $X$ with fixed endpoints. Then there exists an arc $\sigma$ in the class $s$ whose transverse measure is equal to the infimum of the transverse measure of the set of arcs in the class $s$.

Proof (Sketch.) The existence of $\sigma$ can be proved using the same techniques of existence of such a minimizing arc in the case where $X$ is a surface (that is, in the case where $X$ has no singular edges), as exposed in [3], Chapter 5. We note that such an $\operatorname{arc} \sigma$ is quasi-transverse to $F$ in the following sense: either $\sigma$ is a subset of the leaves of $F$, or $\sigma$ is transverse to $F$ and avoids the singularities, or $\sigma$ is a union of embedded arcs which are transverse to $F$, whose endpoints (if they are distinct from those of $\sigma$ ) are on the singular points and such that any two such arcs which are adjacent at a singular point of $F$ are not contained (locally) in the same sector.

There is a basic example of a measured foliation on $X$, which is associated to an ideal hyperbolic structure on $X$. Before giving the definition, we consider the special case where $X$ consists of one single triangle.

Let $T$ be a hyperbolic ideal triangle. Then there is a canonical measured foliation on $T$, called the horocyclic foliation. This is a partial measured foliation of $T$ (that is, a measured foliation whose support is a closed subset of $T$ ), whose leaves are pieces of horocycles which are perpendicular to the edges of $T$ and such that the transverse measure induced on each edge of $T$ coincides with the 1-dimensional Lebesgue measure induced from the hyperbolic metric. The complement of the support of the foliation is a triangular region at the centre of the triangle $T$, bounded by three pieces of horocycles (see Figure 3).

If $X$ is now a 2-dimensional simplicial complex equipped with an ideal hyperbolic structure $h$, then, we can equip each face of $X$ with its canonical horocyclic foliation. The union of these foliations defines a partial measured foliation on $X$. Furthermore, in each face of $X$, we can collapse the unfoliated region onto a tripod (as in Figure 4), and we obtain a measured foliation on $X$ in the sense of Definition 4.1 above and which is called the horocyclic foliation of $X$. To see that this is the case, the only nontrivial fact to prove is that condition (v) of Definition 4.1 above is satisfied, and this is a consequence of the following:

Proposition 4.3. Let h be an ideal hyperbolic structure on $X$. Then, its associated horocyclic measured foliation is trivial around each vertex of $X$.

Proof. Let $S$ be the set of singular points of $X$. By definition, the ideal hyperbolic structure $h$, as a metric on $X-S$, is complete. For each vertex $v$ of $X$, we consider 


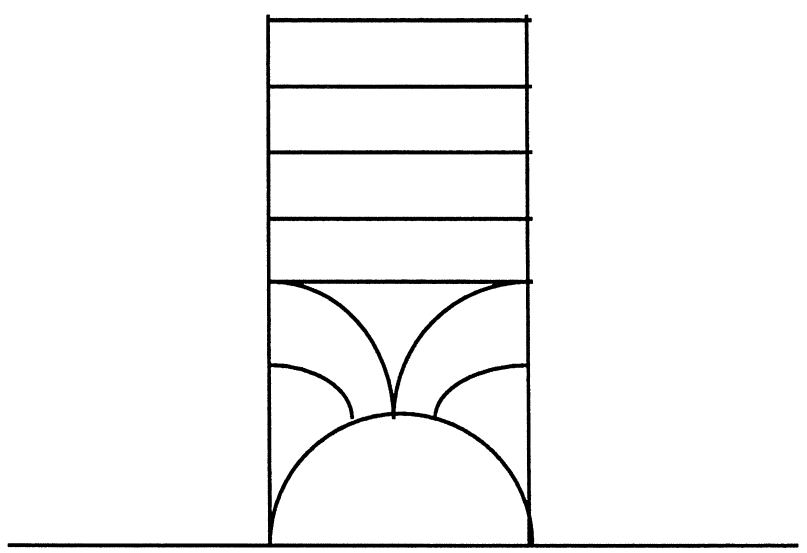

Figure 3

again its link, and we represent it by a simplicial graph $\Gamma(v)$, as we did in Section 3 above. Each simplicial simple closed curve $L \subset \Gamma(v)$ defines a surface $X(L)$ embedded in a neighborhood of $v$, equipped with a hyperbolic structure. The vertex $v$ is a cusp, since the hyperbolic structure in the neighborhhod of $v$ is complete. We already noted that the fact that $v$ is a cusp is equivalent to the fact that the horocyclic foliation around this cusp is a foliation by closed leaves.

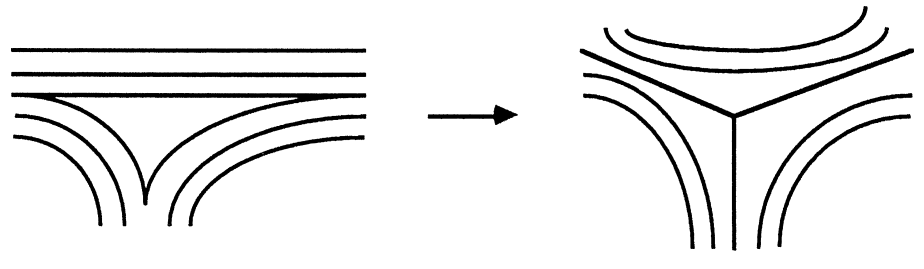

Figure 4

The horocyclic measured foliation on $X$, obtained from the partial horocyclic foliation by collapsing, in each ideal triangle, each nonfoliated region onto a tripod, is well-defined up to equivalence, and the corresponding element in $\mathcal{M F}(X)$ is denoted by $H(h)$.

We now describe a set of parameters for the space $\mathcal{M F}(X)$.

Consider the set $\mathcal{C}$ of triangles and the set $\mathcal{F}$ of gluing maps that define the simplicial complex $X$ and let $F$ be an element of $\mathcal{M F}(X)$. Each triangle in $\mathcal{C}$ is then equipped with a measured foliation induced by $F$, and each edge of this triangle has a distinguished point, which is defined as the intersection of that edge with the leaf joining that edge to the unique singular point in that triangle.

We equip now each edge of $X$ with the fixed orientation that we have chosen at the beginning of Section 2. Let $f: A \rightarrow B$ be an element of $\mathcal{F}$, where $A$ and $B$ are as usual edges of triangles in $\mathcal{C}$. Given the element $F \in \mathcal{M F}(X)$, the edges $A$ and $B$ are equipped with canonical coordinate systems, defined by the transverse measure induced by $F$ on the edge $\pi(A)=\pi(B)$ of $X$, together with the orientation induced by that of the edge $\pi(A)=\pi(B)$ and with the distinguished points on $\pi(A)$ and $\pi(B)$.

In this way, we can associate to each $f \in \mathcal{F}$ a well-defined parameter $y_{F}(f)=$ $y(f) \in \mathbb{R}$, which is called the shift parameter associated to $f$ by $F$. This parameter is 
defined as the algebraic measure of the point $f(a)$, measured in the coordinate system of $B$.

We consider now the shift parameter $y_{F}(f)$ and we compare it with the shift parameter $x_{h}(f)$ which has been defined at the beginning of section 2, associated to a hyperbolic structure $h$ on $X$.

Proposition 4.5. Let $X$ be a 2-dimensional simplicial complex equipped with an ideal hyperbolic structure $h$ and let $F=H(h) \in \mathcal{M F}$ be the equivalence class of the horocyclic foliation of $h$. Then for every gluing map $f: A \rightarrow B$ in $\mathcal{F}$, we have $x_{h}(f)=y_{F}(f)$.

Proof. From the construction, it suffices to note the fact that if $T$ is an ideal hyperbolic triangle equipped with its canonical horocyclic foliation, then the coordinate systems that we have defined on each edge of $X$, associated respectively to the ideal hyperbolic structure and to the horocyclic measured foliations (that is, the metric on the edges, the orientations and the choice of distinguished points on these edges), coincide. This proves the proposition.

We can also reverse the construction:

Proposition 4.6. Let $X$ be a 2-dimensional simplicial complex and let $F \in \mathcal{M F}(X)$. Then, there exists a unique ideal hyperbolic structure $h$ on $X$ such that $F=H(h)$.

Proof. Let $T$ be a triangle in $X$. Then $F$ induces a measured foliation $F_{\mid T}$ on $T$ which is well-defined up to an isotopy whose restriction on the boundary of the triangle is the identity map. Properties (i) to (iii) of Definition 4.1 imply that there exists a unique hyperbolic structure on $T$ which makes this triangle an ideal hyperbolic triangle whose canonical horocyclic foliation is in the equivalence class of the foliation $F_{\mid T}$ after collapsing the non-foliated region of the canonical horocyclic foliation onto a tripod, as in Figure 4 above. (This construction is due to Thurston, see [7] where this idea is used in the case where $X$ is a surface.) The hyperbolic structures on all the triangles of $X$ can be glued together, by using the shift parameters associated to the measured foliation as shift parameters associated to the ideal hyperbolic structure. The result is an ideal hyperbolic structure on $X$, whose horocyclic foliation is in the class of $F$ (Proposition 4.5).

We define now a map $\mathcal{J}: \mathcal{M F}(X) \rightarrow \mathbb{R}^{\mathcal{F}}$ by the formula

$$
\mathcal{J}(F)(f)=y_{F}(f)=y(f),
$$

for all $F \in \mathcal{M F}(X)$ and for all $f \in \mathcal{F}$.

The map $\mathcal{J}$ is injective since each element $F \in \mathcal{M F}(X)$ is completely determined by the set of gluing parameters $y_{F}(f)$, for all $f \in \mathcal{F}$.

We now define the topology of $\mathcal{M F}(X)$ by taking the discrete topology on $\mathcal{F}$ and the product topology on $\mathbb{R}^{\mathcal{F}}$, and by taking on $\mathcal{M F}(X)$ the topology induced by the injective map $\mathcal{J}: \mathcal{M F}(X) \rightarrow \mathbb{R}^{\mathcal{F}}$.

Let $H: \mathcal{T}(X) \rightarrow \mathcal{M F}(X)$ be the map which associates to each element $h \in \mathcal{T}(X)$ the equivalence class of its horocyclic foliation, $H(h)$. 
COROLlary 4.7. The map $H: \mathcal{T}(X) \rightarrow \mathcal{M F}(X)$ is a homeomorphism.

Proof. By Proposition 4.6, the map $H$ is bijective. By Proposition 4.5, this map induces the identity map on the level of parameters. Therefore, $H$ is bicontinuous.

We can use this result to find the dimension of the space $\mathcal{M F}(X)$ in the case where $X$, as in Theorem 3.3, is a finite 2-dimensional simplicial complex. We obtain from Theorem 3.3 and Corollary 4.7 the following

TheORem 4.8. Let $X$ be a finite 2-dimensional simplicial complex with vertices $v_{1}, \ldots, v_{n}$, such that for every $i=1, \ldots, n$, a neighborhood of the vertex $v_{i}$ is the cone over a graph $\Gamma_{i}$ such that the rank of $\pi_{1}\left(\Gamma_{i}\right)$ is equal to $r_{i}$. Let $N$ be the cardinality of the set $\mathcal{F}$ of independent gluing maps. Then, we have

$$
\operatorname{dim} \mathcal{M F}(X)=N-\sum_{i=1}^{n} r_{i}
$$

Let us note that Theorem 4.8, which is a consequence of Corollary 4.7 and Theorem 3.3, can also be proved directly using the same outline of proof as that of Theorem 3.3.

The following is an analogue of Proposition 2.2, and it can also be proved in exactly the same way:

Proposition 4.9. Let $X$ be a 2-dimensional simplicial complex and let $p: \tilde{X} \rightarrow X$ be a covering space of $X$. There is a natural embedding $v: \mathcal{M F}(X) \rightarrow \mathcal{M F}(\tilde{X})$.

5. A parameter space for $\mathcal{T}(X) \cup \mathcal{M F}(X)$. Let $X$ be a 2-dimensional simplicial complex, with $\mathcal{C}$ its associated set of triangles (as in Definition 1.1). We can regard $\mathcal{C}$ as the set of faces of $X$, since there is a natural one-to-one correspondence between these two sets. Let us choose a point in each face of $X$ and let us call it the distinguished point in that face. We note right away that in the case where $X$ is equipped with a hyperbolic structure, so that each face of $X$ is isometric to an ideal hyperbolic triangle, then we shall take the distinguished point in each face to be the centre of that triangle. Similarly, in the case where $X$ is equipped with a measured foliation, we shall take the distinguished point in each face to be the unique singular point of the restriction of the foliation to that face.

Let $X$ be now a 2-dimensional simplicial complex equipped with a set of distinguished points associated to its faces. We define a finite set, which we denote by $\mathcal{S}$, whose elements are certain isotopy classes of arcs joining distinguished points of $X$. These classes are defined in the following way:

Consider a vertex $v$ of $X$ and let $L$ be a simplicial simple closed curve in the link of $v$ (the link is defined in Section 3 above). Let $T_{1}, \ldots, T_{n}$ be the set of triangles which are associated to the edges of $L$, with the cyclic order preserved (we recall that a link of a vertex is a section through $X$ and that the edges of a link are the images of triangles under this section). We can isotop $L$ to a simple closed curve $L^{\prime}$ which contains the distinguished points of the triangles $T_{1}, \ldots, T_{n}$. Then, an element $s \in \mathcal{S}$ is the isotopy class of a subarc of $L^{\prime}$ having its two endpoints on two distinguished points of the sequence of the triangles $T_{1}, \ldots, T_{n}$. Thus, in particular, the isotopy 
class $s$ contains simple arcs. We note also that the endpoints of an arc representing $s$ are distinct except in the case where $s$ can be represented by an arc which coincides with $L^{\prime}$ as a subset of $X$. The set $\mathcal{S}$ is the set of all such elements $s$, for the various vertices of $X$ and the various curves $L$ associated to such a vertex. It is clear from the definition that if $X$ is a finite simplicial complex, then the set $\mathcal{S}$ is finite.

We define now a map $I: \mathcal{T}(X) \rightarrow \mathbb{R}_{+}^{\mathcal{S}}$.

Let $h \in \mathcal{T}(X)$. We define the map $I(h): \mathcal{S} \rightarrow \mathbb{R}_{+}$by taking, for every $s \in \mathcal{S}$, $I(h)(s)$ to be the length of the unique geodesic arc (up to parametrization) in the homotopy class with fixed endpoints $s$ (see Corollary 1.6). As we said above, we are supposing now that the endpoints of the geodesic arc are the centres of the corresponding ideal hyperbolic triangles.

The map $I: \mathcal{T}(X) \rightarrow \mathbb{R}_{+}^{\mathcal{S}}$ is then the map which associates to each element $h \in \mathcal{T}(X)$ the map $I(h): \mathcal{S} \rightarrow \mathbb{R}_{+}$.

We define now in a similar way a map $J: \mathcal{M F}(X) \rightarrow \mathbb{R}_{+}^{\mathcal{S}}$.

Let $F \in \mathcal{M F}(X)$. We define $J(F): \mathcal{S} \rightarrow \mathbb{R}_{+}$by taking, for every $s \in \mathcal{S}, I(h)(s)$ to be the infimum of the total transverse measure of an arc in the homotopy class with fixed endpoints $s$. Proposition 4.2 says that there exists a curve realizing the infimum and that therefore the infimum is a minimum. Again, as we said above, we are taking here the endpoints of such an arc at the singular points of the foliation $F$ which are contained in the triangles defined by the homotopy class $s$.

The map $J: \mathcal{T}(X) \rightarrow \mathbb{R}_{+}^{\mathcal{S}}$ is then the map which associates to each element $F \in \mathcal{M F}(X)$ the map $J(F): \mathcal{S} \rightarrow \mathbb{R}_{+}$.

We equip the space $\mathcal{S}$ with the discrete topology and the space $\mathbb{R}_{+}^{\mathcal{S}}$ with the product topology.

The main result of this section is the following

THEOREM 5.1. For every 2-dimensional simplicial complex $X$, each of the two maps $I: \mathcal{T}(X) \rightarrow \mathbb{R}_{+}^{\mathcal{S}}$ and $J: \mathcal{M} \mathcal{F}(X) \rightarrow \mathbb{R}_{+}^{\mathcal{S}}$ is a homeomorphism onto its image. Furthermore, in the case where where $X$ has at least one vertex whose link is not simply connected, then the images $I(\mathcal{T}(X))$ and $J(\mathcal{M F}(X))$ are disjoint.

As we noted above, if $X$ is a finite 2-dimensional simplicial complex, then the set $\mathcal{S}$ is finite, which implies that in this case, $\mathbb{R}_{+}^{\mathcal{S}}$ is finite dimensional.

We begin by showing that the map $I: \mathcal{T}(X) \rightarrow \mathbb{R}_{+}^{\mathcal{S}}$ is continuous. This will be a consequence of the following more general result.

Let $\tilde{X}$ be the universal cover of $X$ and let $T$ and $T^{\prime}$ be two arbitrary faces of $\tilde{X}$. We define the map $\ell_{(.)}\left(T, T^{\prime}\right): \mathcal{T}(X) \rightarrow \mathbb{R}_{+}$which to every element $h \in \mathcal{T}(X)$ associates the quantity $\ell_{h}\left(T, T^{\prime}\right)$, equal to distance $h^{\prime}\left(P, P^{\prime}\right)$ in $\tilde{X}$. (Here, $h^{\prime}$ is the lift of the metric $h$ on the universal cover $\tilde{X}$, and $P^{\prime}$ and $P$ are respectively the centres of the triangles $T$ and $T^{\prime}$.) We have then the following:

Proposition 5.2. The map $\ell_{(.)}\left(T, T^{\prime}\right): \mathcal{T}(X) \rightarrow \mathbb{R}_{+}$is continuous.

Proof. Consider the centres $P$ and $P^{\prime}$ of the faces $T$ and $T^{\prime}$ respectively. Since $\tilde{X}$ is simply connected, the distance $h^{\prime}\left(P, P^{\prime}\right)$ is equal to the length of the unique local geodesic joining these points. The image of this geodesic crosses a certain sequence $T_{1}, \ldots, T_{k}$ of consecutive faces of $\tilde{X}$ (with $T_{1}=T$ and $T_{k}=T^{\prime}$ ). By Proposition 1.9, the union $T_{1} \cup \ldots \cup T_{k}$ is isometric to an ideal hyperbolic polygon $X_{0}$ in $\mathbb{H}^{2}$, whose isometry type is completely determined by the shift parameters associated to the 
sequence of edges joining adjacent pairs of triangles, $\left(T_{i}, T_{i+1}\right)(i=1, \ldots, k)$. We can see now that the distance $h^{\prime}\left(P, P^{\prime}\right)$, clearly varies continuously in terms of these shift parameters. This proves Proposition 5.2.

COROLlaRy 5.3. The map $I: \mathcal{T}(X) \rightarrow \mathbb{R}_{+}^{\mathcal{S}}$ is continuous.

Proof. Consider again the universal cover $\tilde{X}$ of $X$ and let $c$ be an arc representing a homotopy class $s \in \mathcal{S}$. Consider a lift $\tilde{c}$ of $c$ to $\tilde{X}$ and let $T$ and $T^{\prime}$ be the triangles in $\tilde{X}$ containing the endpoints of $\tilde{X}$. For every $h \in \mathcal{T}(X)$, the value $I(h)(s)$ is equal to the distance in $\tilde{X}$ between the centres of $T$ and $T^{\prime}$. The continuity of the map $I$ follows therefore from Proposition 5.2.

The proof of the injectivity of the map $I$, which we give now, is more involved than the proof of the continuity.

Proposition 5.4. The map $I: \mathcal{T}(X) \rightarrow \mathbb{R}_{+}^{\mathcal{S}}$ is injective.

Proof. Let $\mathcal{F}$ be the set of gluing maps of $X$ (as in Definition 1.1). Let $h$ and $h^{\prime}$ be two elements in $\mathcal{T}(X)$, with $I(h)=I\left(h^{\prime}\right)$. We wish to show that this implies $h=h^{\prime}$. For that purpose, it suffices to prove the equality $x_{h}(f)=x_{h^{\prime}}(f)$ for every element $f \in \mathcal{F}$. The proof of this fact is divided into several lemmas:

Consider a hyperbolic ideal quadrilateral obtained by gluing two ideal triangles $T$ and $T^{\prime}$, using a map $f: A \rightarrow A^{\prime}$ where $A$ (respectively $A^{\prime}$ ) is and edge of $T$ (respectively of $T^{\prime}$ ). The image of $A$ (respectively of $A^{\prime}$ ) in the quadrilateral is a diagonal, which we call $D$. We choose an orientation on $D$ so that the value of the shift $x(f)$ is well-defined. It is clear that the value of that shift determines the isometry type of the quadrilateral. Let $P$ and $P^{\prime}$ be the centres of the triangles $T$ and $T^{\prime}$ respectively.

Lemma 5.5. The absolute value of the shift parameter, $|x(f)|$, is determined by the distance $d\left(P, P^{\prime}\right)$.

Proof. By elementary hyperbolic geometry, the distance $d\left(P, P^{\prime}\right)$ between the two centres is a convex function of the shift parameter $x(f)$. There is a minimum for $d\left(P, P^{\prime}\right)$ at the point where the shift is zero. By symmetry, two different shift parametrs corresponding to the same distance $d\left(P, P^{\prime}\right)$ are equal in absolute value (see Figure 5). This proves Lemma 5.5.

COROLlary 5.6.The equality $I(h)=I\left(h^{\prime}\right)$ implies that for every element $f \in \mathcal{F}$, the shift parameters $x_{h}(f)$ and $x_{h^{\prime}}(f)$ have the same absolute value.

Proof. It suffices to note that by Lemma 5.5, for every $f \in \mathcal{F}$, the absolute value $\left|x_{h}(f)\right|$ is determined by $I(h)(s)$, with $s$ being the homotopy class with fixed endpoints of a curve joining the centres of two adjecent triangles in $X$.

To continue the proof of Proposition 5.4, we let $v$ be a vertex of $X$ and $L$ a simplicial simple closed curve in the link of $v$. We orient all the half-edges of $X$ which correspond to the vertices of $L$ so that they point towards $v$. We choose an orientation on $L$ and we denote the faces of $X$ which correspond to the vertices of $L$ by 

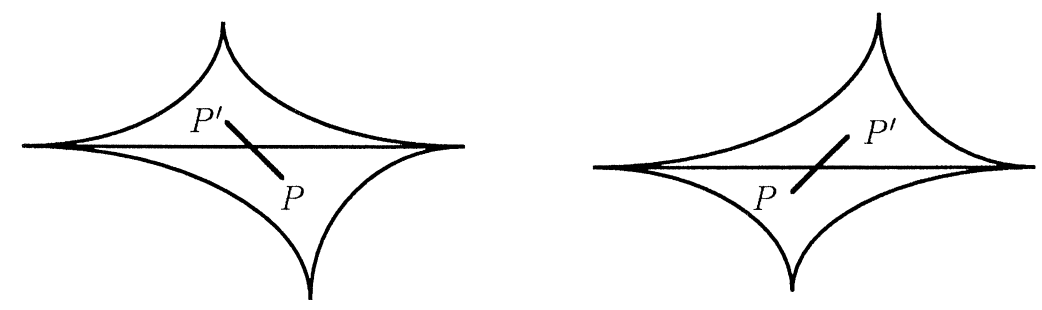

Figure 5

$T_{1}, \ldots, T_{n}$ with the cyclic order preserved. Given an ideal hyperbolic structure on $X$, we have in this way, for any two adjacent faces $\left(T_{i}, T_{i+1}\right)$, a well-defined gluing map $f_{i}$ which is an isometry between edges of ideal hyperbolic triangles, and a welldefined value of the shift, $x\left(f_{i}\right)$ (we are making the convention that $T_{n+1}=T_{1}$ ).

Lemma 5.7. The shift values $x\left(f_{1}\right), \ldots, x\left(f_{n}\right)$ cannot all have the same sign, except in the case where $x\left(f_{i}\right)=0$ for all $i=1, \ldots, n$.

Proof. This follows from Proposition 3.2, which gives the fact that the hyperbolic structure on $X$ is complete implies that $x\left(f_{1}\right)+\ldots+x\left(f_{n}\right)=0$. From that follows easily the proof of Lemma 5.7.

Lemma 5.8. Consider the two ideal hyperbolic structures $h$ and $h^{\prime}$ on $X$ satisfying $I(h)=I\left(h^{\prime}\right)$, and let $x_{h}\left(f_{1}\right), x_{h}\left(f_{2}\right), x_{h^{\prime}}\left(f_{1}\right)$ and $x_{h^{\prime}}\left(f_{2}\right)$ be the shift parameters for the gluing maps associated to the pairs of consecutive triangles $\left(T_{1}, T_{2}\right)$ and $\left(T_{2}, T_{3}\right)$. Then, the following cases are excluded:

(i) $x_{h}\left(f_{1}\right)<0, x_{h}\left(f_{2}\right) \geq 0, x_{h^{\prime}}\left(f_{1}\right)>0$ and $x_{h^{\prime}}\left(f_{2}\right) \leq 0$;

(ii) $x_{h}\left(f_{1}\right)<0, x_{h}\left(f_{2}\right)>0, x_{h^{\prime}}\left(f_{1}\right)<0$ and $x_{h^{\prime}}\left(f_{2}\right)<0$;

(iii) $x_{h}\left(f_{1}\right)<0$ and $x_{h}\left(f_{2}\right)>0, x_{h^{\prime}}\left(f_{1}\right)>0$ and $x_{h^{\prime}}\left(f_{2}\right)>0$.

Proof. Let $P_{1}, P_{2}$ and $P_{3}$ be the centres of $T_{1}, T_{2}$ and $T_{3}$ respectively, for the metric $h$, and let $P_{1}^{\prime}, P_{2}^{\prime}$ and $P_{3}^{\prime}$ be the centres of the same triangles, for the metric $h^{\prime}$. The three triangles are represented in Figures 6, 7 and 8. In these figures, we have represented a portion of the universal cover of the space $X$ and we are studying lifts of the triangles $T_{1}, T_{2}$ and $T_{3}$. We denote these lifts with the same letters. The cusp $v$ is represented as the point at infinity in the upper-half space model of $\mathbb{H}^{2}$. From Corollary 5.6, the shift parameters have the same absolute value. There is a monotone symmetric relationship between the shift parameter and the signed angle at the center of the triangle between the perpendicular to the side and the arc between centers of adjacent triangles. This angle lies in the interval $(-\pi / 3, \pi / 3)$. Let $\theta_{j}$ (respectively $\theta_{j}^{\prime}$ ) be the angle corresponding to $x_{h}\left(f_{j}\right)$ (respectively $x_{h^{\prime}}\left(f_{j}\right)$ ). Let $\beta$ be the angle facing infinity at $P_{2}\left(\right.$ or $\left.P_{2}^{\prime}\right)$ of the arc $P_{1} P_{2} P_{3}$ (respectively $P_{1}^{\prime} P_{2}^{\prime} P_{3}^{\prime}$ ). Then in Figure 6 we have $\beta=2 \pi / 3+\left|\theta_{1}\right|+\left|\theta_{3}\right|$, in Figure 7 we have $\beta=2 \pi / 3-\left|\theta_{1}\right|-\left|\theta_{3}\right|$ and in Figure 8 we have $\beta=2 \pi / 3+\left|\theta_{1}\right|-\left|\theta_{3}\right|$. If $\theta_{1}$ is non-zero, the first two are distinct, if $\theta_{2}$ is non-zero, the first and third are not equal. To prove now that case (i) cannot hold, consider a triangle which is adjacent to $T_{2}$, and which shares with $T_{2}$ the side which is different from the sides adjacent to $T_{1}$ and $T_{3}$ and let $P_{4}$ (respectively $P_{4}^{\prime}$ ) be its centre for the metric $h$ (respectively for the metric $h^{\prime}$ ). (We stress on the fact that we are taking the triangles with center $P_{4}$ and $P_{4}^{\prime}$ to be images of the 
same triangle in the abstract simplicial complex, since there may be many choices of triangles glued to this side of $T_{2}$.) This triangle is represented in Figures 6 and 7 respectively. From the discussion above, the two pairs of triangles $P_{1} P_{2} P_{4}, P_{2} P_{4} P_{3}$ and $P_{1}^{\prime} P_{2}^{\prime} P_{4}^{\prime}, P_{2}^{\prime} P_{4}^{\prime} P_{3}^{\prime}$ cannot be isometric. This proves that case (i) cannot hold. To prove that (ii) cannot hold, we use the same arguments on Figures 6 and 8. Case (iii) is symmetric to case (ii). This proves Lemma 5.8.

We can conclude now the proof of Proposition 5.4. Consider again the vertex $v$, the curve $L$ with its orientation, the sequence of triangles $T_{1}, \ldots, T_{n}$, the gluing maps $f_{1}, \ldots, f_{n}$ associated to the pairs of consecutive triangles, and the two sequences of shift parameters $x_{h}\left(f_{1}\right), \ldots, x_{h}\left(f_{n}\right)$ and $x_{h^{\prime}}\left(f_{1}\right), \ldots, x_{h^{\prime}}\left(f_{n}\right)$. Recall that the goal is to prove that for every $i=1, \ldots, n$, we have $x_{h}\left(f_{i}\right)=x_{h^{\prime}}\left(f_{i}\right)$. By Corollary 5.6, it suffices to prove that for every $i=1, \ldots, n, x_{h}\left(f_{i}\right)$ and $x_{h^{\prime}}\left(f_{i}\right)$ are either zero or have the same sign. Suppose for contradiction that the signs were not all the same. In this case, using Lemma 5.7, we can suppose, without loss of generality, that there exists an

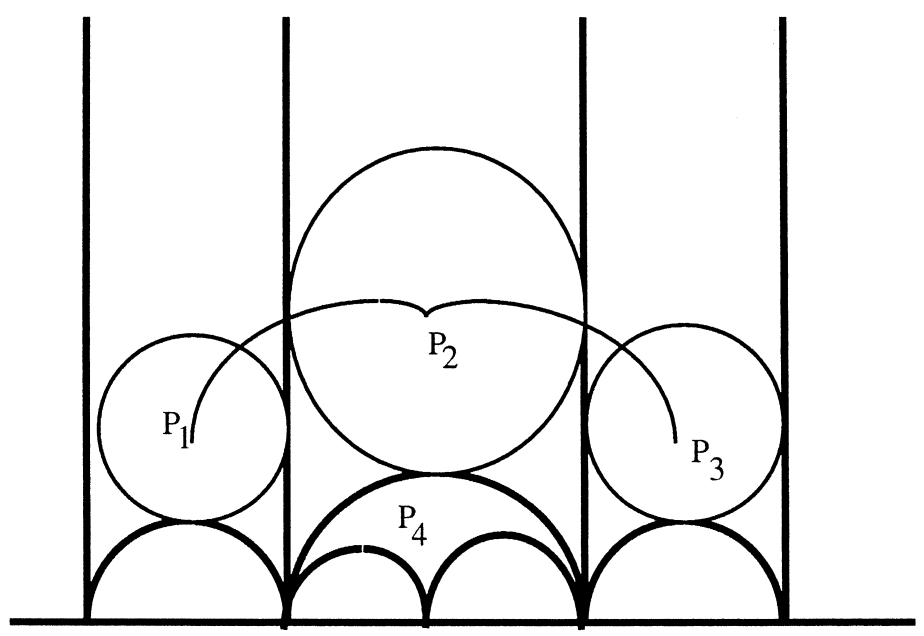

Figure 6

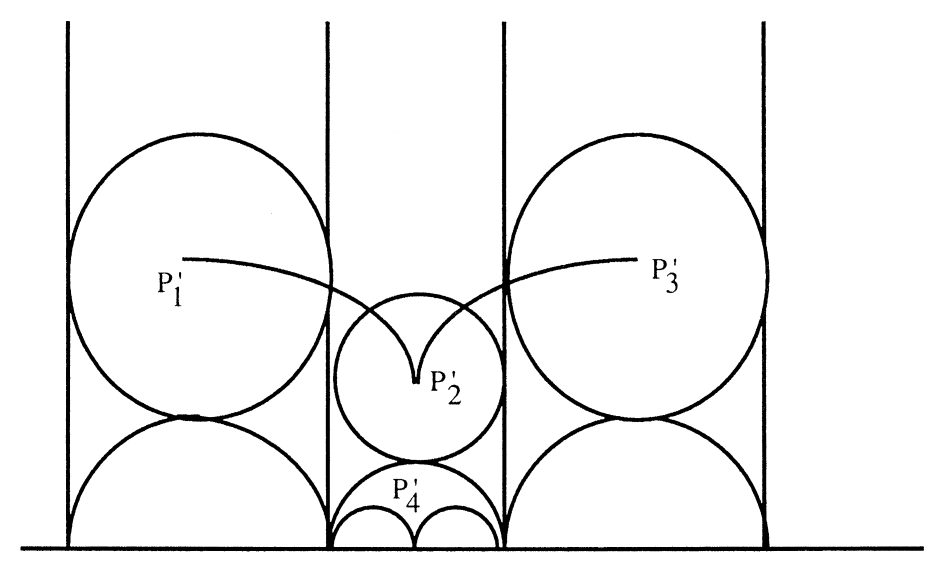

Figure 7 


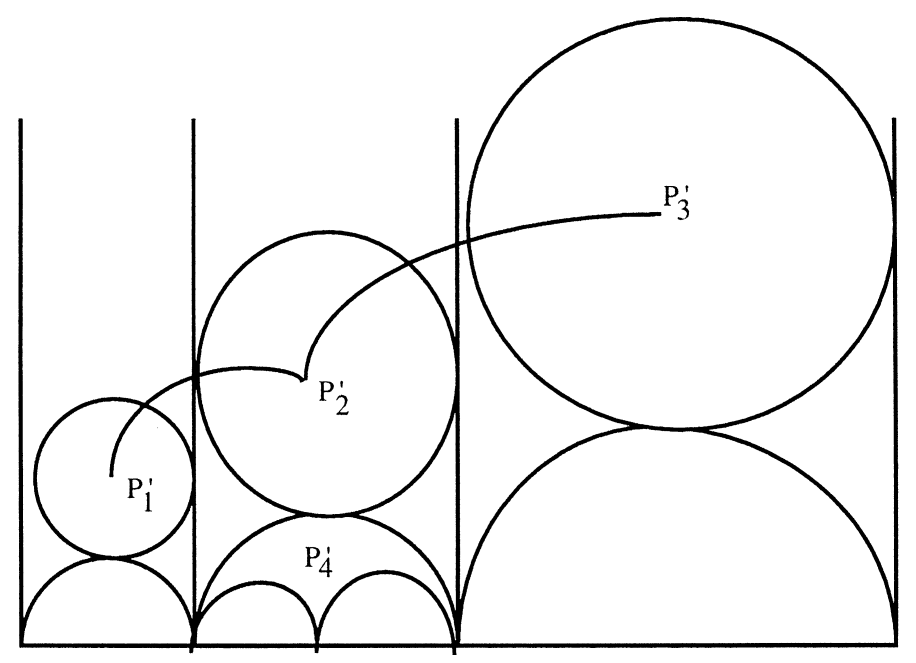

Figure 8

integer $i \in\{1, \ldots, n\}$ such that $x_{h}\left(f_{i}\right)<0$ and $x_{h^{\prime}}\left(f_{i}\right)<0$. It is possible now that the same sequence of signs holds for the index $i+1$, that is, it is possible to have $x_{h}\left(f_{i+1}\right)<0$ and $x_{h^{\prime}}\left(f_{i+1}\right)<0$ (we recall the convention $f_{n+1}=f_{1}$ ). We examine then the index $i+2$ and so on. This process must stop since by Lemma 5.7, the sequence of signs of $x_{h}\left(f_{1}\right), \ldots, x_{h}\left(f_{n}\right)$ cannot be all negative. We arrive then at a sequence of pairs of consecutive triangles $\left(T_{j}, T_{j+1}\right)$ whose gluing maps satisfy one of the following three properties:

(i) $x_{h}\left(f_{j}\right)<0, x_{h}\left(f_{j+1}\right) \geq 0, x_{h^{\prime}}\left(f_{j}\right)>0$ and $x_{h^{\prime}}\left(f_{j+1}\right) \leq 0$;

(ii) $x_{h}\left(f_{j}\right)<0, x_{h}\left(f_{j+1}\right)>0, x_{h^{\prime}}\left(f_{j}\right)<0$ and $x_{h^{\prime}}\left(f_{j+1}\right)<0$;

(iii) $x_{h}\left(f_{j}\right)<0$ and $x_{h}\left(f_{j+1}\right)>0, x_{h^{\prime}}\left(f_{1}\right)>0$ and $x_{h^{\prime}}\left(f_{j+1}\right)>0$.

The three cases are excluded by Lemma 5.7. This implies that for every $i=1, \ldots, n$, we have $x_{h}\left(f_{i}\right)=x_{h^{\prime}}\left(f_{i}\right)$. This completes the proof of Proposition 5.4.

We study now the map $J: \mathcal{M F}(X) \rightarrow \mathbb{R}_{+}^{\mathcal{S}}$, and we begin with the continuity.

We let $\tilde{X}$ be as above the universal cover of $X$, and $T$ and $T^{\prime}$ two arbitrary faces of $\tilde{X}$. Let $m_{(.)}\left(T, T^{\prime}\right): \mathcal{M F}(X) \rightarrow \mathbb{R}_{+}$be the map which to every element $F \in \mathcal{M F}(X)$ associates the quantity $m_{F}\left(T, T^{\prime}\right)$, defined as the infimum of the transverse measure of an arc in $\tilde{X}$ joining the singular points contained in $T$ and $T^{\prime}$. Here, the space $\tilde{X}$ is equipped with the foliation $F^{\prime}$ which is the inverse image of $F$ by the quotient map $\tilde{X} \rightarrow X$. We have then the following:

Proposition 5.9. The map $m_{(.)}\left(T, T^{\prime}\right): \mathcal{M F}(X) \rightarrow \mathbb{R}_{+}$is continuous.

Proof. Let $P$ and $P^{\prime}$ be the singular points of $F$ which are contained respectively in the faces $T$ and $T^{\prime}$. There is an arc $c$ joining $P$ and $P^{\prime}$ whose transverse measure is equal to the infimum of the transverse measure of an arc in $\tilde{X}$ joining these points (Proposition 4.2). This curve crosses a certain number $T_{1}, \ldots, T_{k}$ of consecutive faces of $\tilde{X}$ (with $T_{1}=T$ and $T_{k}=T^{\prime}$ ), and the union $T_{1} \cup \ldots \cup T_{k}$ is a simply connected surface equipped with a measured foliation whose type (as a foliated surface) is completely determined by the shift parameters defined by $F$ and associated to the sequence of edges joining adjacent pairs of triangles, $\left(T_{i}, T_{i+1}\right)(i=1, \ldots, k)$. From 
this, we can see that the total transverse measure of $c$ clearly varies continuously in terms of these shift parameters.

\section{COROLlary 5.10. The map $J: \mathcal{M F}(X) \rightarrow \mathbb{R}_{+}^{\mathcal{S}}$ is continuous.}

Proof. To prove this fact, we let $\tilde{X}$ be as above the universal cover of $X$ and let $c$ be an arc representing a homotopy class $s \in \mathcal{S}$. Consider a lift $\tilde{c}$ of $c$ to $\tilde{X}$, and let $T$ and $T^{\prime}$ be the triangles in $\tilde{X}$ containing the endpoints of $\tilde{c}$. For every $F \in \mathcal{M F}(X)$, the value $J(F)(s)$ is equal to the total transverse measure of an arc in $\tilde{X}$ joining the singular points in $T$ and $T^{\prime}$ for the lifted measured foliation $F^{\prime}$ of $F$. The continuity of the map $J$ follows therefore from Proposition 5.9.

Proposition 5.11. The map $J: \mathcal{M F}(X) \rightarrow \mathbb{R}_{+}^{\mathcal{S}}$ is injective.

Proof. Let $F$ and $F^{\prime}$ be two elements of $\mathcal{M F}(X)$. To prove the proposition, we first note the following

Lemma 5.12. The equality $J(F)=J\left(F^{\prime}\right)$ implies that for every element $f \in \mathcal{F}$, the shift parameters $x_{F}(f)$ and $x_{F^{\prime}}(f)$ have the same absolute value.

Proof. The proof is analogous to that of Corollary 5.6.

The rest of the proof of Proposition 5.11 that we give now is simpler than that of the analogous result for ideal hyperbolic structures (that is, Proposition 5.4). As we did before the statement of lemma 5.7, we let $v$ be a vertex of $X$ and $L$ a simplicial simple closed curve in the link of $v$, equipped with an orientation. We let also $T_{1}, \ldots, T_{n}$ be the faces of $X$ which correspond to the edges of $L$ with the cyclic order preserved, and for each $i=1, \ldots, n$, we let $f_{i}$ be the gluing map between the edges of the consecutive pair of faces $T_{i}$ and $T_{i+1}$ (with the convention $T_{n+1}=T_{1}$ ). To prove proposition 5.11, it suffices now to prove the following

Lemma 5.13. The equality $J(F)=J\left(F^{\prime}\right)$ implies that for every vertex $v$, for every curve $L$ as above in the link of $v$ and for every $i=1, \ldots, n$, we have $x_{F}\left(f_{i}\right)=x_{F^{\prime}}\left(f_{i}\right)$.

Proof. It is convenient for the proof to make an appropriate choice of the sequence of triangles $T_{1}, \ldots, T_{n}$ associated to the curve $L$ (in other words, we are making a choice of the first triangle $T_{1}$, and the indices of the other triangles follow by cyclic order). To see which choice we make, consider, in the neighborhood of $v$, a surface $X_{F}(L) \subset X$ which corresponds to the simple closed curve $L$. The surface $X_{F}(L)$ is a cylinder equipped with a foliation induced by $F$, whose leaves are simple closed curves which are all homotopic between themselves and homotopic to the vertex $v$. We take $X_{F}(L)$ to be the largest such surface. Therefore, there is necessarily a singular point of $F$ on the boundary of that surface (see Figure 9). In fact, there may be several singular points on the boundary of of $X_{L}(F)$ and we make a choice of a singular point on the boundary on $X_{F}(L)$, and we take now $T_{1}$ to be the triangle containing that singular point. We have then the sequence $T_{1}, \ldots, T_{n}$, by using the cyclic order.

Consider now the foliation $F^{\prime}$. We claim that the singular point of $F^{\prime}$ which is contained in the triangle $T_{1}$ is also on the boundary of the corresponding surface $X_{F^{\prime}}(L)$. To see that this is the case, consider again the foliation $F$ and let $s \in \mathcal{S}$ be the 


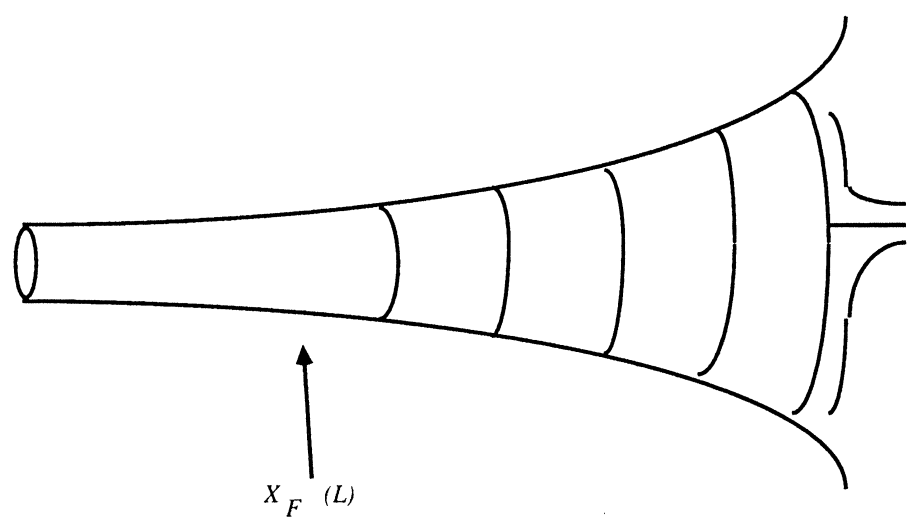

Figure 9

homotopy class of a simple closed curve with fixed endpoints, these endpoints being both at the singular point of $F$ contained in $T_{1}$, and such that the free homotopy class of that curve is equal to the class of the simplicial curve $L$. Then, the condition that the singular point of $F$ in $T_{1}$ is on the boundary of $X_{F}(L)$ is equivalent to the condition $J(F)(s)=0$. Now since $J(F)=J\left(F^{\prime}\right)$, we have $J(F)(s)=0$ if and only if $J\left(F^{\prime}\right)(s)=0$. This proves the claim.

Let $C$ be the boundary curve of the surface $X_{F}(L)$ and let $s_{1}, \ldots, s_{n}$ be now the homotopy classes with fixed endpoints of curves contained in the simplicial closed curve $L$ such that for every $i=1, \ldots, n, s_{i}$ joins the centre of $T_{1}$ to the centre of $T_{i+1}$ (with the convention, as before, that $T_{n+1}=T_{1}$ ). Then, for each $i=1, \ldots, n$, the quantity $J(F)\left(s_{i}\right)$ is equal to the transverse measure $F\left(C_{i}\right)$ of a curve $C_{i}$ contained in the triangle $T_{i}$ which joins the singular point $P_{i}$ to the curve $C$ and which is transverse to the horocyclic foliation (see Figure 10). It is easy to see now by induction that the set of transverse measures $\left\{F\left(C_{i}\right)\right\}$ determines the set of shift parameters $\left\{x_{F}\left(f_{i}\right): i=1, \ldots, n\right\}$. If $F$ and $F^{\prime} \in \mathcal{M F}(X)$ satisfy $J(F)=J\left(F^{\prime}\right)$, then for every such curve $C_{i}$, we have $F\left(C_{i}\right)=F^{\prime}\left(C_{i}\right)$. Therefore, if $J(F)=J\left(F^{\prime}\right)$, we have for every $i=1, \ldots, n, x_{F}\left(f_{i}\right)=x_{F^{\prime}}\left(f_{i}\right)$. This proves Lemma 5.13 .

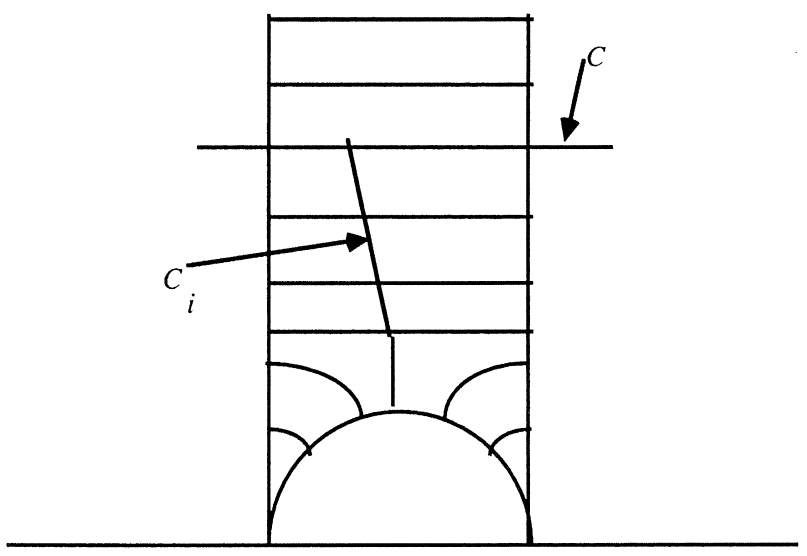

Figure 10 
We can complete now the proof of Theorem 5.1.

By Propositions 5.4 and 5.11 and Corollaries 5.3 and 5.9, the maps $I$ and $J$ are continuous and injective. We have also the following

Lemma 5.14. The maps $I: \mathcal{T}(X) \rightarrow \mathbb{R}_{+}^{\mathcal{S}}$ and $J: \mathcal{M F}(X) \rightarrow \mathbb{R}_{+}^{\mathcal{S}}$ are proper.

Proof. We prove that $I$ is proper, the proof that $J$ is proper being similar.

Since $I$ is injective, this map is proper if and only if it is a closed map. To prove that $I$ is a closed map, let $K \subset \mathcal{T}(X)$ be a closed subset and let us prove that $K^{\prime}=I(K)$ is a closed subset of $\mathbb{R}_{+}^{\mathcal{S}}$. For this, we shall prove that the closure $\bar{K}^{\prime}$ of $K^{\prime}$ is contained in $K^{\prime}$.

Let $y \in \bar{K}^{\prime}$. Then, there exists a sequence $y_{n} \in K^{\prime}$ with $y_{n} \rightarrow y$. We want to prove now that $y \in K^{\prime}$. Let $h_{n} \in K$ such that $I\left(h_{n}\right)=y_{n}$.

If some subsequence $\left(h_{n_{k}}\right)$ of the sequence $\left(h_{n}\right)$ converges to some $h \in \mathcal{T}(X)$, then $h \in K$ since $K$ is closed. From the continuity of $I$, we have then $I(h)=y$, which shows that $y \in K^{\prime}$.

If there is no such a subsequence $h_{h_{k}}$, then the sequence $\left(h_{n}\right)$ is not bounded (recall that $\mathcal{T}(X)$ is homeomorphic to a Euclidean space $\mathbb{R}^{k}$ ). We prove now that this leads to a contradiction. Indeed, let $h_{n}=\left(x_{n}^{1}, \ldots, x_{n}^{k}\right)$ under the homeomorphism which identifies $\mathcal{T}(X)$ with $\mathbb{R}^{k}$, where for each $i$ and $n, x_{n}^{i}$ is the shift parameter of an isometry $f: A \rightarrow B$ where $A$ and $B$ are edges of two triangles $T$ and $T^{\prime}$ which are consecutive in $X$ (see Section 3 above). Since the sequence $\left(h_{n}\right)$ is unbounded, there exists a "coordinate sequence" (or subsequence of ) $x_{n}^{i}$ such that $x_{n}^{i} \rightarrow \infty$ as $n \rightarrow \infty$. Let $P$ and $P^{\prime}$ denote the centres of the triangles $T$ and $T^{\prime}$ respectively. Since $x_{n}^{i} \rightarrow \infty$ as $n \rightarrow \infty$, we have $\ell_{n}\left(P, P^{\prime}\right) \rightarrow \infty$, where $\ell_{n}\left(P, P^{\prime}\right)$ denotes the length, with respect to $h_{n}$, of the geodesic segment which joins $P$ and $P^{\prime}$ in the union of the two faces $T$ and $T^{\prime}$. From that we deduce that the sequence $\left(y_{n}\right)$ cannot converge to $y$, which contradicts our hypothesis. This proves that the map $I$ is proper.

By the theorem of the invariance of domain, each of the maps $I$ and $J$ is a homeomorphism onto its image. To complete the proof of Theorem 5.1, it remains to show that the images of $I$ and $J$ are disjoint in the case where there exists at least one vertex of $X$ whose link is not simply connected.

Let $h$ be an ideal hyperbolic structure on $X$ and let $s \in \mathcal{S}$. Then $I(h)(s)$ is strictly positive. Indeed, this quantity is bounded below by $2 \times$ the distance of the centre of an ideal triangle to the boundary of that triangle. If now $F$ is a measured foliation on $X$, then we can find an element $s \in \mathcal{S}$ such that $J(F)(s)=0$. Indeed, choose a vertex $v$ of $X$ whose link is not simply connected. Since $F$ is trivial around $v$, there exists a maximal cylinder equipped with a foliation induced by $F$, which is a foliation by circles centred at $v$. On the boundary of that cylinder, there is a singular point (or several singular points) of $F$ (see Figure 9), and there is a compact leaf $L$ which is a circle containing this singular point (respectively a closed interval having its endpoints at two such singular points). The homotopy class with fixed endpoints of that leaf $L$ is an element $s \in \mathcal{S}$ which satisfies $J(F)(s)=0$. This completes the proof of Theorem 5.1.

6. The boundary of the Teichmüller space of a 2-dimensional simplicial complex. Let $X$ be a 2-dimensional simplicial complex. We have seen that if $X$ is a 2-dimensional simplicial complex, there is a natural homeomorphism $H: \mathcal{T}(X) \rightarrow \mathcal{M F}(X)$ 
(Corollary 4.7). In the case where $X$ is finite, then the spaces $\mathcal{T}(X)$ and $\mathcal{M F}(X)$ are both homeomorphic to $\mathbb{R}^{k}$, where the value of $k$ is defined in terms of the combinatorics of $X$. We wish to relate in a more geometric manner the two spaces $\mathcal{T}(X)$ and $\mathcal{M F}(X)$. We assume that $X$ has at least one vertex whose link is not simply connected (as in Theorem 5.1).

There is a special point in $\mathcal{T}(X)$ (respectively in $\mathcal{M F}(X)$ ), which we shall call the zero-ideal hyperbolic structure (respectively the zero- measured foliation, or measured foliation class), defined by the fact that the shift parameter associated to any two adjacent faces is zero. (Note that the zero measured foliation is the image by the map $H$ of the zero-ideal hyperbolic structure, and that there is indeed an ideal hyperbolic structure and a measured foliation satisfying the required properties since the relation $(*)$ for completeness, defined in Section 3, satisfied for every vertex $v$ ). Let us denote by $\{0\}$ the zero-measured foliation class. It has the property that all the regular leaves are closed leaves around a vertex.

Let $\mathcal{P} \mathcal{M F}(X)$ be the set of rays in $\mathcal{M F}(X)-\{0\}$, that is, the quotient of this space by the obvious action of the set of positive real numbers $\mathbb{R}_{+}$and let $\pi: \mathcal{M F}(X) \rightarrow \mathcal{P} \mathcal{M F}(X)$ be the quotient map. With the identification $H$ above between $\mathcal{T}(X)$ and $\mathcal{M F}(X)$, the space $\mathcal{T}(X)$ is also equipped with an action of $\mathbb{R}_{+}$, and we can therefore talk about rays in that space. Thus, we can always realize the space $\mathcal{P} \mathcal{M} \mathcal{F}(X)$ as a boundary of $\mathcal{T}(X)$, by attaching to each ray in $\mathcal{T}(X)$ an endpoint, which is its projective class, considered as an element of $\mathcal{P} \mathcal{M F}(X)$.

The two embeddings $J: \mathcal{T}(X) \rightarrow \mathbb{R}_{+}^{\mathcal{S}}$ and $I: \mathcal{M F}(X) \rightarrow \mathbb{R}_{+}^{\mathcal{S}}$ allow us to realize this bordification in a more natural way, which generalizes Thurston's compactification of the Teichmüller space of a surface. Indeed, in the special case where our 2dimensional simplicial complex is a punctured surface, the boundary of Teichmüller space that we obtain here agrees with the boundary described in [6], which is Thurston's boundary (as is it proved in [6]).

In the rest of this section, we consider a finite 2-dimensional simplicial complex $X$. The space $\mathbb{R}_{+}^{\mathcal{S}}$ is finite dimensional in this case, since the set $\mathcal{S}$ is finite. We consider the set of rays in $\mathbb{R}_{+}^{\mathcal{S}}$, which we denote by $P R_{+}^{\mathcal{S}}$, and which is defined as the quotient of $R_{+}^{\mathcal{S}}-\{0\}$ by the natural action of the set $\mathbb{R}_{+}$of positive real numbers. We have then a natural compactification $R_{+}^{\mathcal{S}} \cup P R_{+}^{\mathcal{S}}$ of $R_{+}^{\mathcal{S}}$ by adjoining to each ray its point at infinity. The space $\mathcal{M F} \cup \mathcal{P} \mathcal{M F}$ is naturally embedded in the compact space $R_{+}^{\mathcal{S}} \cup P R_{+}^{\mathcal{S}}$.

We can now study the embedding $\mathcal{M F} \cup \mathcal{P} \mathcal{M F} \subset R_{+}^{\mathcal{S}} \cup P R_{+}^{\mathcal{S}}$. Theorem 6.2 below gives a criterion for the convergence of a sequence of points in $\mathcal{M F}$ to a point in $\mathcal{P} \mathcal{M F}$. This theorem generalizes a result which is valid for surfaces with cusps (see [6], Theorem 3.7), and it is proved using the same outline. We start with the following lemma, which is analogous to the "Fundamental lemma" of ([3], p. 143)

Lemma 6.1. There exists a constant $C>0$ such that for every $s \in \mathcal{S}$ and for every $h \in \mathcal{T}(X)$, we have

$$
J(H(h))(s) \leq I(h)(s) \leq J(H(h))(s)+C,
$$

where $H(h)$ denotes as before the equivalence class of the horocyclic foliation of $h$.

Proof. Since the set $\mathcal{S}$ is finite, it suffices to prove that for every $s \in \mathcal{S}$, there is a constant $C=C(s)>0$ such that for every $h \in \mathcal{T}(X)$, the two inequalities in (6.1.1) 
are satisfied. We shall prove this fact with $C=4 i(s, \Lambda)$, the minimum number of intersection points of a curve in the class $s$ with the set $\Lambda$ of edges of $X$. Let us fix therefore an element $s \in \mathcal{S}$ and let $X$ be equipped with an ideal hyperbolic structure $h$. By Corollary 1.6, we can represent $s$ (in a unique way) by a curve $\sigma$ in $X$, having its endpoints at the centres of two triangles, such that $\sigma$ is a local geodesic for the metric $h$. Let $\sigma_{1}, \ldots, \sigma_{n}$ denote the successive intersections of $\sigma$ with the set $\operatorname{Supp}(H(h))-\Lambda$, where $\operatorname{Supp}(F)$ denotes the support of a foliation $F$ and where $\Lambda$ denotes as before the set of edges of the 2-dimensional simplicial complex $X$. Each $\sigma_{i}$ is a piece of geodesic whose type of intersection with an ideal triangle is of one of the types (a), (b) and (c) indicated in Figure 11. These three types are as follows:

type (a): $\sigma_{i}$ has its two endpoints on $\Lambda$

type (b): $\sigma_{i}$ has only one endpoint on $\Lambda$

type (a): $\sigma_{i}$ has no endpoint on $\Lambda$.

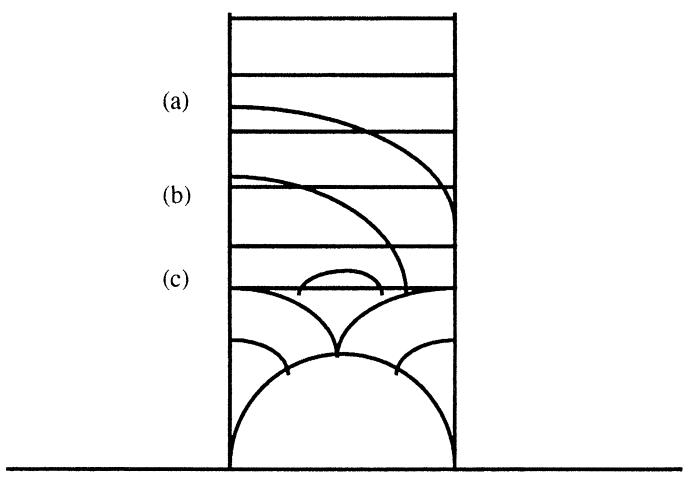

Figure 11

Note that since $\sigma$ is geodesic, each of the segments $\sigma_{i}$ has at most one point of tangency with the horocyclic foliation. We now make the following modification on the curve $\sigma$ (we note that after the modification, $\sigma$ will no more be geodesic).

For each $i=1, \ldots, n$, we make each segment $\sigma_{i}$ which is of type (a) or (b) transverse to the horocyclic foliation, while keeping its endpoints fixed, and we push each segment $\sigma_{i}$ of type (c) outside the support of $H(h)$, in the central nonfoliated triangle contained in the ideal triangle containing $\sigma_{i}$. Let $\sigma_{i}^{\prime}$ be the segment obtained in this way from of $\sigma_{i}$, and let $\sigma^{\prime}$ be the curve obtained from $\sigma$ by replacing each segment $\sigma_{i}$ by $\sigma_{i}^{\prime}$.

For every hyperbolic structure $g$ on $X$, for every measured foliation $M$ on $X$ and for every curve $c$ transverse to $F$, let us denote by $L_{g}(c)$ the length of $c$ with respect to the metric $g$ and by $F_{M}(c)$ the transverse measure of $c$ with respect to $M$.

Consider now a segment $\sigma_{i}^{\prime}$ which is not contained in the complement of $\operatorname{Supp}(H(h))$. We have $F_{H(h)}\left(\sigma_{i}^{\prime}\right) \leq L_{h}\left(\sigma_{i}\right)$ since $F_{H(h)}\left(\sigma_{i}^{\prime}\right)$ is equal, by definition of the transverse measure, to the length of an orthogonal projection of $\sigma_{i}^{\prime}$ on the boundary of the ideal triangle to which it belongs. Taking the sum over all these pieces of $\sigma^{\prime}$, we obtain $F_{H(h)}\left(\sigma^{\prime}\right) \leq L_{h}(\sigma)$, which implies $J(H(h))(s) \leq I(h)(s)$, which is the first inequality in (6.1.1).

We prove now the second inequality in (6.1.1). For that purpose, we represent now the homotopy class $s$ by a curve $\tau$ which is quasi-transverse to the foliation $H(h)$ and which satisfies therefore the relation 


$$
J(H(h))(s)=F_{H(h)}(\tau)
$$

(see Proposition 4.2). In particular, every connected component of the intersection of $\tau$ with $\operatorname{Supp}(H(h))$ is an arc which is transverse to $H(h)$.

Without loss of generality, we can suppose that the curve $\tau$ has minimal intersection (with respect to its isotopy class) with the set $\Lambda$ of edges of $X$. Indeed, by general position, we can first suppose that $\tau$ is transverse to $\Lambda$. If $\operatorname{card}(\tau \cap \Lambda)>i(s, \Lambda)$, then there exists a disk $D$ which is embedded in $X$ such that $\partial D$ is the union of an arc in $\tau$ and an arc in $\Lambda$ (see Corollary 2.4 in [2]). The foliation induced by $H(h)$ on $D$ is of one of the two types described in figure 12 (a) and (b). In each case, we can push the curve $\tau$ along the leaves of $H(h)$, while keeping $\tau$ quasitransverse to $H(h)$, and strictly decreasing the quantity $\operatorname{card}(\tau \cap \Lambda)$. Thus, we can suppose that $\operatorname{card}(\tau \cap \Lambda)=i(s, \Lambda)$.

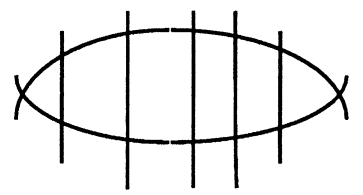

(a)

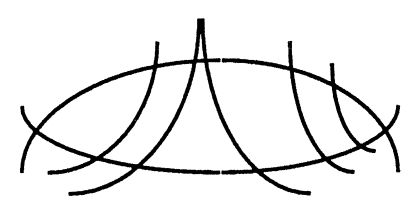

(b)

Figure 12

Let $\tau_{1}, \ldots, \tau_{n}$ denote the connected components of the intersection of $\tau$ with the set $\operatorname{Supp}(H(h))-\Lambda$, and let $\tau_{1}^{\prime}, \ldots, \tau_{m}^{\prime}$ denote the connected components of the intersection of $\tau$ with $X-\operatorname{Supp}(H(h))$.

For each $i=1, \ldots, m$, the component $\tau_{i}^{\prime}$ is contained in a small non-foliated region in an ideal triangle. We replace this segment, keeping its enpoints fixed, by a geodesic segment, which we still call $\tau_{i}^{\prime}$. The diameter of the nonfoliated region in an ideal hyperbolic triangle being bounded by 1 , the length of each $\tau_{i}^{\prime}$ is bounded by 1 . Since the number of components $\tau_{i}^{\prime}$ is bounded by $2 \times i(s, \Lambda)$, we can write

$$
\sum_{i=1}^{m} L_{g}\left(\tau_{i}^{\prime}\right) \leq 2 \times i(s, \Lambda) .
$$

For each $i=1, \ldots, n$, we replace the segment $\tau_{i}$ by an arc which is the union of a piece of horocycle $h_{i}$ contained in the ideal triangle containing $\tau_{i}$ and of a geodesic segment $g_{i}$ contained in $\Lambda$, as in Figure 13 .

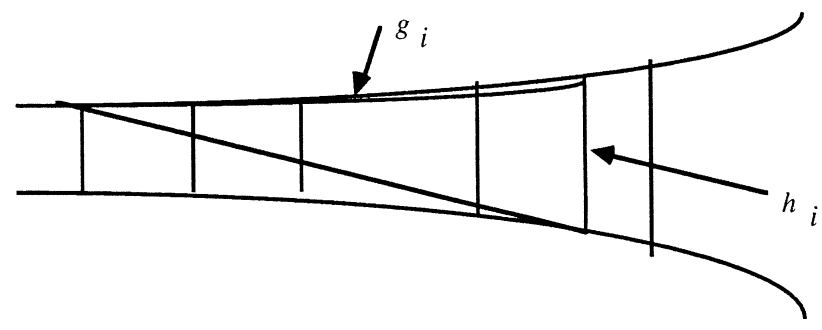

Figure 13 
We have therefore:

$$
L_{g}\left(g_{i}\right)=F_{H(h)}\left(g_{i}\right)=F_{H(h)}\left(\tau_{i}\right)
$$

The number of arcs of the form $h_{i}$ is bounded above by $2 i(s, \Lambda)$ and the length of each of these arcs is bounded above by the length of the largest leaf of the horocyclic foliation of an ideal hyperbolic triangle, which is equal to 1 . Therefore, we have

$$
\sum_{i=1}^{n} L_{g}\left(h_{i}\right) \leq 2 i(s, \Lambda) .
$$

If $\tau^{\prime \prime}$ is now the curve obtained from $\tau$ by replacing each arc $\tau_{i}$ by the corresponding arc $h_{i} \cup g_{i}$, then we have, by (6.1.2), (6.1.3) and (6.1.4),

$$
L_{g}\left(\tau^{\prime \prime}\right) \leq F_{H(h)}\left(\tau^{\prime \prime}\right)+4 i(s, \Lambda) .
$$

Since $F_{H(h)}\left(\tau^{\prime \prime}\right)=F_{H(h)}(\tau)$, we obtain

$$
L_{g}\left(\tau^{\prime \prime}\right) \leq F_{H(h)}(\tau)+4 i(s, \Lambda)
$$

Therefore, we have

$$
I(h)(s) \leq J(H(h))(s)+4 i(s, \Lambda),
$$

which completes the proof of Lemma 6.1.

We obtain now the following

THEOREM 6.2. Let $h_{n}$ be a sequence of points in $\mathcal{T}(X)$ tending to infinity in that space (that is, this sequence eventually leaves any compact subset of $\mathcal{T}(X)$ ). Then $h_{n}$ converges to a point in $\mathcal{P} \mathcal{M F}(X)$ if and only if the sequence of horocyclic foliations $H\left(h_{n}\right)$ converges projectively (that is, in the space $\mathcal{P} \mathcal{M F}(X)$ ), and in this case the two sequences converge to the same point.

Proof. Using Lemma 6.1 above, the proof of Theorem 6.2 can be done in the same way as the proof of Corollary II.3 of [3], Chapter 1, using the "Fundamental Lemma".

\section{REFERENCES}

1. C. Charitos and A. Papadopoulos, The homeomorphisms of 2-dimensional simplicial complexes (paper in preparation).

2. C. Charitos and G. Tsapogas, Complexity of geodesics on 2-dimensional ideal polyhedra and isotopies, Math. Proc. Camb. Phil. Soc. 121 (1997), 343-258.

3. A. Fathi, F. Laudenbach and V. Poenaru, Travaux de Thurston sur les surfaces, Astérisque 66-67 (1979). 
4. E. Ghys and P. de la Harpe (Ed.) Sur les groupes hyperboliques d'après Mikhael Gromov, Progress in Mathematics, 83. (Birkhäuser Boston, Inc., Boston, 1990).

5. M. Gromov, Structures métriques pour les variétés riemanniennes (rédigé par J. Lafontaine et P. Pansu), (Fernand Nathan, Paris, 1981).

6. A. Papadopoulos, Sur le bord de Thurston de l'espace de Teichmüller d'une surface non compacte, Math. Ann. 282 (1988), 353-359.

7. W. P. Thurston, Some stretch maps between hyperbolic surfaces, preprint, 1984.

8. W. P. Thurston, Three-dimensional geometry and Topology, Vol. 1, (Edited by Silvio Levy), Princeton Mathematical Series 35, 1997. 